\title{
Detecting Variability in Massive Astronomical Time-series Data. III. Variable Candidates in the SuperWASP DR1 Found by Multiple Clustering Algorithms and a Consensus Clustering Method
}

\author{
Min-Su Shin ${ }^{1}$, Seo-Won Chang ${ }^{2,3}$, Hahn $\mathrm{Yi}^{4}$, Dae-Won Kim ${ }^{5}$, Myung-Jin Kim ${ }^{1}$, and Yong-Ik Byun ${ }^{6}$ \\ ${ }^{1}$ Korea Astronomy and Space Science Institute, 776, Daedeokdae-ro, Yuseong-gu, Daejeon 34055, Republic of Korea; msshin@kasi.re.kr \\ ${ }^{2}$ Research School of Astronomy and Astrophysics, The Australian National University, Canberra, ACT 2611, Australia; seowon.chang@anu.edu.au \\ ${ }^{3}$ ARC Centre of Excellence for All-sky Astrophysics (CAASTRO), Building A28, School of Physics, The University of Sydney, Sydney, NSW 2006, Australia \\ ${ }^{4}$ Asan Medical Center, 88, Olympic-ro 43-Gil, Songpa-gu, Seoul 05505, Republic of Korea; hahn.yi@gmail.com \\ ${ }^{5}$ Electronics and Telecommunications Research Institute, 218 Gajeong-ro, Yuseong-gu, Daejeon, 34129, Republic of Korea; dwkim78@gmail.com \\ ${ }^{6}$ Department of Astronomy, Yonsei University, 50 Yonsei-ro, Seodaemun-gu, Seoul 03722, Republic of Korea; skarma@kasi.re.kr, ybyun@yonsei.ac.kr \\ Received 2016 August 8; revised 2018 September 5; accepted 2018 September 16; published 2018 October 17
}

\begin{abstract}
We determine candidate variable sources in the SuperWASP Data Release 1 (DR1) using multiple clustering methods and identifying variable candidates as outliers from large clusters. We extract 15,788,814 light curves that have more than 15 photometric measurements in the SuperWASP DR1. Variations in the light curves are described in terms of nine variability features that are complementary to each other. We consider three different clustering methods based on Gaussian mixture models, including one that was used in our previous work, assuming that real variable candidates can be found as minor clusters and at a distant from major clusters, which correspond to nonvariable objects. The three different methods with a broad level of speed and precision prove that we can select a suitable method for detecting variable light curves, depending on the speed and precision constraints on clustering. We also consider a consensus clustering method that combines clustering results obtained using multiple clustering methods. The consensus clustering method improves the reliability of detecting variable candidates by combining information that is learned from a given data set by multiple methods. As a complete variability analysis of the public SuperWASP light curves, we provide clustering results obtained by using an infinite Gaussian mixture model in the framework of variational Bayesian inference, as well as variability indices of the light curves in an online database to help others exploit the SuperWASP data.
\end{abstract}

Key words: astronomical databases: miscellaneous - methods: data analysis - methods: statistical - stars: variables: general

\section{Introduction}

Public releases of massive time-series data have been proven to be important for making observation data collected in wide-field surveys available to a broad range of astronomy communities, for a wide range of science goals. These publicly available data can be used as a reference for comparing to future observations. Different analysis methods can be used, exploring different classes of variable sources that have not been considered or detected by original projects. Some examples of time-series observation projects with public data releases are the Massive Compact Halo Object Project (Alcock et al. 1993), Optical Gravitational Lensing Experiment (Udalski et al. 1992), All Sky Automated Survey (Pojmanski 1997), Northern Sky Variability Survey (NSVS; Woźniak et al. 2004), Lincoln NearEarth Asteroid Research (Sesar et al. 2011), and Catalina Surveys (Drake et al. 2009).

One interesting analysis that uses publicly available timeseries data amounts to using novel variability detection methods that are different from the methods adopted by the original survey projects. The reliable detection of variable candidates is the first step toward investigating the physics of variable sources. Moreover, because follow-up observations of interesting variable candidates can become expensive as the number of variable candidates increases, estimating the reliability of variability detection is likely to become important in future large surveys such as the Large Synoptic Survey Telescope (LSST; Ivezic et al. 2008).
We proposed a novel approach that uses clustering methods with density estimation to detect variable candidates in massive astronomical time-series data in our previous papers (Shin et al. 2009, 2012). The proposed method can be considered as a complementary method to classical conventional methods. In the classical approach, the properties of light curves are simulated or assumed for a given setup of observations, and then statistical cuts are determined to obtain the best detection results of variable sources (e.g., Price-Whelan et al. 2014). When statistical distributions of a variability statistic are analytically well known, an analytic cut of variable candidates is commonly adopted. Because variability signals in time-series data are distorted and vary owing to factors such as different variability types, observation patterns, and photometric qualities, a good method of variability detection should account for these factors.

Using clustering methods and finding variable candidates as objects that are located in minor clusters and are far from major clusters can genuinely group into large clusters non-variable objects and objects that are dominantly affected by these common systematic effects or for which variable signals are too weak. Therefore, this approach does not require well-defined prior knowledge of hidden variable sources, systematic problems associated with the data, and observation setups, enabling machine learning algorithms to be used for learning the properties of the given data from the data. Moreover, our method does not assume a specific statistical distribution (e.g., a Gaussian distribution) for treating non-variable light curves 
(see Grubbs 1969 for a discussion of fundamental issues). Naturally, variable candidates are identified as outliers separate from major groups of light curves, and our approach becomes more powerful on larger data sets that allow our machine learning algorithms to better learn the properties of nonvariable light curves.

There are two major limitations to using a single clustering method for detecting variable sources. First, clustering methods, being one class of unsupervised machine learning methods, do not possess a good intrinsic criterion for validating the results of clustering, and these methods correspond to fundamentally ill-posed problems. In variability detection using supervised learning methods, it is possible to validate machine learning models on validation data sets that consist of data with known properties of variability signals (e.g., Brink et al. 2013). However, clustering methods do not have preexisting known validation test data and preexisting correct solutions for checking the validity of clustering results (see Jain 2010 for a review). These effects owing to the lack of validation data sets can be alleviated by employing multiple different clustering methods to improve the reliability and validity of clustering results (Jain et al. 2004). In general, different clustering methods will yield different clustering results when applied to the same data set. Thus, if several different clustering methods group the same object as a member of minor clusters, the object is likely to be a reliable variable candidate. Second, some clustering methods are computationally too expensive to be used for frequently updated large data sets and repeated real-time applications of clustering methods. For example, the non-parametric Bayesian clustering method used in our previous papers utilizes a Markov Chain Monte Carlo with a Gibbs sampler, which is generally computationally expensive when being used on large multidimensional data sets, and is generally the slowest step in an entire analysis process (Bishop 2007; Marin 2007). Fast clustering can be adopted for a quick analysis of growing data sets collected in big surveys such as LSST (Ridgway et al. 2014) in addition to processing the final data set using a slow clustering method, although fast clustering methods might have disadvantages compared to slow clustering methods.

In this paper, we present a new strategy for detecting variable candidates in massive astronomical time-series data by using a variational approximate Bayesian inference that is much faster than the Markov Chain Monte Carlo method. We also demonstrate how to practically combine clustering results from multiple clustering methods with different properties and computational steps. To exemplify the method's applicability, we present results from consensus clustering (also called ensemble clustering, being part of ensemble methods), which infers the best clustering result by considering agreements among the results obtained using several different clustering results (see Zhou 2012 for a review). Although ensembling multiple results together can make strongly variable light curves easily distinguishable, it can also mix weakly variable light curves with clusters of non-variable light curves as objects of disagreement among the different clustering methods. Therefore, we intend to use consensus clustering to increase the contrast between definitely variable and non-variable light curves in the clustering results.

Following the methodology used in our two previous papers (Shin et al. 2009, 2012), we adopt Gaussian mixture models (Reynolds 2015) to generate clustering structures that become inputs to the ensemble clustering method. Using the same clustering models allows us to apply the ensemble method for the NSVS data included in the previous papers and to try a combined analysis of the NSVS data and the different data examined in this paper together for common objects between the two data sets. We add some new features describing information of the training data to those used in the previous papers, making it easy to update the variability indices of the NSVS data for combined analysis in the future.

We choose as an analysis data set the first data release of the Wide Angle Search for Planets (WASP) project (Butters et al. 2010). The WASP project is a wide-field imaging survey performed using SuperWASP detectors of multiple $200 \mathrm{~mm}$ lenses and $2 k \times 2 k$ CCDs (Pollacco et al. 2006). Although the primary scientific goal of the project is finding transiting exoplanets, the data generated by the project can be used for different scientific studies in time-domain astrophysics (Norton 2012). In particular, rich information on variable stars has been explored for some specific types of variables in the database (e.g., Norton et al. 2007; Hebb et al. 2010; Thomas et al. 2010; Norton et al. 2011; Smalley et al. 2011; McQuillin et al. 2012; Schaffenroth et al. 2013). Thus, our detection of all kinds of variable candidates can help people explore general kinds of variable sources that are likely to be present in the WASP data set that contains about 30 million objects.

The variability analysis of WASP data is also useful because most objects in the data are optically sufficiently bright to be detected by telescopes with small telephoto lenses, and they are already included in preexisting multiwavelength data. As we explored with the NSVS data, our detection of variability in light curves can be combined with the knowledge of spectral energy distributions produced by surveys such as IRAS (Clegg 1980), AKARI (Murakami et al. 2007), WISE (Wright et al. 2010), Two Micron All Sky Survey (2MASS; Kleinmann 1992), and GALEX (Bianchi \& GALEX Team 1999; Martin et al. 2005), allowing the identification of specific variability processes such as mass-losing late-type stars or RR Lyrae stars.

The paper is organized as follows. In Section 2, we explain the light curves in the WASP data and the variability indices derived from the light curves. Our clustering methods are explained in Section 3, demonstrating how to combine results obtained using multiple clustering methods and the process of combining results in reliable clustering. In Section 4, we describe a method for extracting variable candidates by using the results of clustering. Variable candidates are examined in Section 5, considering their associations with other data generated by multiwavelength observations. Finally, the summary and discussion are presented in the last section.

\section{Data and Variability Indices}

\subsection{Preprocessing of Data}

The basic data set consists of light curves that are included in the first data release of the WASP project (Butters et al. 2010). ${ }^{7}$ The original data contain 17,970,999 light curves constructed from 3,631,972 images. Because light curves with few data points (i.e., observations) are not useful, we select light curves that have more than 15 data points, resulting in $15,884,797$

\footnotetext{
7 The data are now available in http://wasp.cerit-sc.cz/ (Paunzen et al. 2014) or http://exoplanetarchive.ipac.caltech.edu/applications/ExoTables/search.html? dataset=superwasptimeseries, where the way to access the data and the class of the data is different from the original archive that we accessed.
} 
light curves (i.e., about $88 \%$ of the original data set), as done in our previous paper (Shin et al. 2012, hereafter Paper II). If we adopt 30 as the minimum number of data points in the raw light curves, the total number of acceptable light curves becomes $15,371,664$ (i.e., about $86 \%$ of the original data set). These light curves are spatially divided into $10^{\circ} \times 10^{\circ}$ fields along the R.A. and decl. directions. Overall, 319 fields cover the distribution of the 15,884,797 light curves in the sky.

We first group the light curves into multiple sets ${ }^{8}$ that share similar time-sampling patterns. The WASP project has frequently changed its observation strategy, such as its observation fields and its instrumental setups, such as the types of filters (Pollacco et al. 2006; Butters et al. 2010). Moreover, the same patch of the sky could be observed by both the northern and southern sites of the WASP project, each utilizing different observation strategies, different time windows, and different cameras. Because all of these factors can affect the systematic properties of light curves in the data set, we have to separate out similar groups of light curves sharing similar observation properties.

To resolve the issue of variable observation properties, we group light curves into a single set when these light curves have similar starting and ending times. By finding the largest change in the variance of the starting time $\left(t_{\mathrm{s}}\right)$, ending time $\left(t_{\mathrm{e}}\right)$, and number of data points for light curves in a group, we find that the best cut is $\sqrt{\left(\delta t_{\mathrm{s}}{ }^{2}+\delta t_{\mathrm{e}}{ }^{2}\right)}<37 \mathrm{~s}$ for two light curves with the time differences of $\delta t_{\mathrm{s}}$ and $\delta t_{\mathrm{e}}$. When we use a small time difference as a cut value, the number of groups increases, reducing the variance. Meanwhile, using a large time difference as a similarity cut yields an insufficient number of groups, with large internal variance. The adopted cut value is the one that produces optimal separation.

Because we adopt no constraints on the sky positions, it is possible that the step of constructing sets can make some light curves be in the same group based on similar time-sampling patterns, although their sky positions might not be close. As an example, Figure 1 shows the largest set of light curves that are found to be irregularly distributed in the sky. Because systematic patterns hidden in light curves can change over a large range of spatial scales, it is not surprising to find a strong variation of light curves even among spatially close light curves. We note that our clustering results are presented with spatial distributions of cluster members in the sky for helping to identify spatially distinct patterns that might not represent light curves of real variable sources, as we show in Section 4.

Our clustering analysis, described in what follows, uses only 6478 sets, each containing more than 60 light curves. Therefore, the final data set for our clustering analysis contains $15,795,861$ light curves after dropping the light curves not grouped together due to large time differences. Because the minimum number cut of 60 light curves is arbitrarily chosen, our online release of the analysis results still includes the rest of the light curves although they are not included in the clustering analysis. Others are welcome to try different clustering methods, including the light curves that are not included in our clustering analysis.

\footnotetext{
8 We use the term "set" to mean the collection of light curves with similar time-sampling patterns while the term "field" is used to represent spatially close groups of light curves in the sky.
}

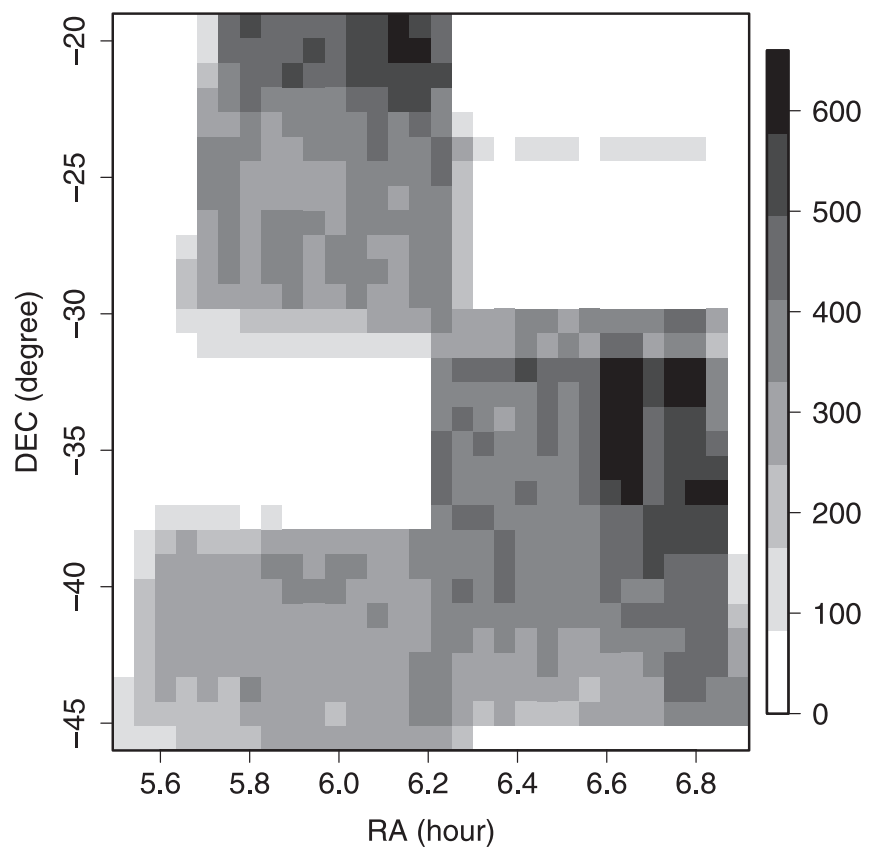

Figure 1. Spatial distribution of the largest set found together with \left.${\sqrt{\left(\delta t_{\mathrm{s}}\right.}}^{2}+\delta t_{\mathrm{e}}^{2}\right)<37$. The total number of light curves is 199,113 over R.A. 5.493 to $6.919 \mathrm{hr}$ and decl. $-46.983^{\circ}$ to $-20^{\circ}$. This number histogram contains $30 \times 30$ same-size bins.

\subsection{Variability Indices}

We use nine variability indices that describe patterns of light variation in the light curves to project the raw time-series data onto nine-dimensional feature space. Table 1 summarizes the variability indices that are estimated from the flux measurements for given light curves. This combination of indices is similar to that used in our previous papers (Shin et al. 2009, 2012; see references therein). $\sigma / \mu$ and $\sigma / \nu$ are the ratio of dispersion over the mean and median, respectively. $\gamma_{1}$ and $\gamma_{2}$ denote the skewness and kurtosis as simple descriptive statistics, respectively. Con measures how many series of three consecutive measured values deviate more than $2 \sigma$ from the median value. Basically, Con is similar to the Runs test statistic for detecting non-randomness (Wald \& Wolfowitz 1940). $\eta$ is the von Neuman ratio, which is a small-sample version of the Durbin-Watson statistic for testing autocorrelation in the observed values (i.e., non-randomness) (Habibagahi \& Pratschke 1972). $J$ and $K$ have been commonly used in astronomy to detect non-random pattern in the light curves (Stetson 1996). AoVM represents the strength of periodic signal found in periodograms derived from the analysis of the variance statistic.

A slight modification of these indices is not likely to have a significant effect, considering the fact that the indices complement each other. In addition to the indices used in our previous work, we include $\sigma / \nu$ in this paper. If all light curves are satisfactorily described by Gaussian random variates and the observational time sampling does not produce biased light curves, these multiple variability indices are not needed to find meaningful light variations in the light curves. However, because systematic sampling patterns and hidden systematic patterns are common in the data, we do not expect the distributions of these indices to be a simple one. In general, multivariate treatment of data is necessary to maximize the precision of extracting a small fraction of suspected 
Table 1

Variability Indices

\begin{tabular}{|c|c|}
\hline Index & Definition \\
\hline $\begin{array}{l}\sigma / \mu \\
\sigma / \nu \\
\gamma_{1}\end{array}$ & $\begin{array}{l}\text { Weighted sample standard deviation/weighted sample mean } \\
\text { Weighted sample standard deviation/median } \\
\qquad \frac{\sqrt{N(N-1)}}{N-2} \frac{\sum_{n=1}^{N}\left(x_{n}-\mu\right)^{3} / N}{\sqrt[3]{\sum_{n=1}^{N}\left(x_{n}-\mu\right)^{2} / N}}\end{array}$ \\
\hline$\gamma_{2}$ & $\frac{N-1}{(N-2)(N-3)}\left\{(N+1)\left(\frac{\sum_{n=1}^{N}\left(x_{n}-\mu\right)^{4} / N}{\left(\sum_{n=1}^{N}\left(x_{n}-\mu\right)^{2} / N\right)^{2}}-3\right)+6\right\}$ \\
\hline Con & $1+\frac{1}{N-2} \sum_{n=1}^{N-2}\left\{\begin{aligned} 1 \quad \text { if }\left(x_{n}-\nu\right) & >2 \sigma \text { and }\left(x_{n+1}-\nu\right) \\
& >2 \sigma \text { and }\left(x_{n+2}-\nu\right) \\
& >2 \sigma \\
1 \quad \text { if }\left(x_{n}-\nu\right) & <2 \sigma \text { and }\left(x_{n+1}-\nu\right) \\
& <2 \sigma \text { and }\left(x_{n+2}-\nu\right) \\
0 & <2 \sigma \\
0 & \text { otherwise }\end{aligned}\right.$ \\
\hline$\eta$ & $\frac{\sum_{n=1}^{N-1}\left(x_{n+1}-x_{n}\right)^{2} /(N-1)}{\sigma^{2}}$ \\
\hline $\begin{array}{l}J \\
K\end{array}$ & $\begin{array}{c}\sum_{n=1}^{N-1} \operatorname{sign}\left(\delta_{n} \delta_{n+1}\right) \sqrt{\left|\delta_{n} \delta_{n+1}\right|} \\
\frac{1 / N \sum_{n=1}^{N}\left|\delta_{n}\right|}{\sqrt{1 / N \sum_{n=1}^{N} \delta_{n}^{2}}}\end{array}$ \\
\hline
\end{tabular}

AoVM The maximum value of the analysis of variance (ANOVA) statistic (Schwarzenberg-Czerny 1989, 1996)

Note. $\gamma_{1}$ and $\gamma_{2}$ are the skewness and kurtosis of $N$ fluxes $x_{n}$, respectively (see Joanes \& Gill 1998; Rimoldini 2014 for other possible estimations). $\delta_{n}$ is $\sqrt{N /(N-1)}\left(x_{n}-\mu\right) / e_{n}$, where $e_{n}$ is a photometric flux error for each data point. $\operatorname{Sign}\left(\delta_{n} \delta_{n+1}\right)$ is the sign of $\delta_{n} \delta_{n+1}$. We compute AoVM by using a public code provided in http://astromsshin.github.io/science/code/MultiStep_Period/ (Shin \& Byun 2004).

patterns from a very large set of light curves (Bhat 2011). If a specific parametric model of light curves such as microlensing and transit models is a main concern and adequate, parameters describing the parametric light curve model can be considered as variability indices, different from the simple descriptive indices adopted here (e.g., Nieto-Barajas \& Contreras-Cristán 2014).

The main purpose of using AoVM is to detect and measure strongly periodic signals in light curves, whether or not these signals represent non-physical processes such as observation time sampling. Our estimation of AoVM might not be the best way to derive the correct strength of variability signals in light curves over a correct period because various methods can be used with a broad range of periodicity model parameters with a choice of statistics (see Thieler et al. 2013 for discussions). However, because we adopt a fixed model and its model parameters homogeneously for all light curves, the distribution of AoVM values does not introduce artificial effects into a given set of light curves that are influenced by similar observing patterns.

We drop problematic measurements of fluxes stored in the original DR1 light curves. First, data points with negative fluxes are not included in the estimation of the variability indices. Second, whenever two data points are reported at the same observation time (owing to simultaneous observations by multiple cameras mounted together), we use only the second measurement in the original light curve. Because a single object can be observed by two cameras mounted together, the light curve of the object can feature two simultaneously acquired data points. In this case, we use only one data point.
Because any systematic differences between two simultaneously acquired data points cannot be easily resolved without consulting the original data, we adopt the simple rule of using only one data point. We note that using the average of the multiple measurements instead of choosing a single measurement works only if they do not have any systematic effects and are completely independent. We expect that future research with a different methodology can be considered for this case of multiple simultaneous measurements. After this screening step, we obtain 15,865,772 light curves, each with more than 15 data points.

We derive the variability indices from raw calibrated photometric measurement instead of using detrended data. The DR1 data include two sets of flux measurements (Butters et al. 2010). One set contains the raw calibrated flux values that are obtained after performing a standard photometric process (Kane et al. 2004). The other set includes the flux values obtained after detrending of the systematic patterns found in the light curves (Collier Cameron et al. 2006). In order to avoid and isolate the effects of this detrending on our clustering analysis, we do not use detrended fluxes.

Figure 2 shows the distribution of the nine variability indices of 29,770 example light curves. The distribution does not follow the expected pattern of the normal distribution, which has $\gamma_{1} \sim 0$ and $\gamma_{2} \sim 0$. The broad distribution of the variability indices implies the existence of multiple subpopulations in the data dominated by different types of signals, systematic patterns, and random noise. $\sigma / \mu$ and $\sigma / \nu$ should be close to each other under the assumption of normal distribution for observed fluxes. However, we find a deviation of $\sigma / \nu$, which we newly include as a variability index compared to our previous paper (Shin et al. 2012), from the distribution of $\sigma / \mu$.

In practice, it is computationally challenging to derive variability indices from a large number of light curves. The index estimation procedure consists of processing thousands of flux measurements per light curve. After testing two configurations of parallel distributed processing using the Condor system $^{9}$ (Thain et al. 2005) and the Hadoop environment ${ }^{10}$ (see Wiley et al. 2011 for an example in astronomy) with its streaming processing (Kwak et al. 2011), we choose the Condor system as our computing environment to calculate the variability indices of all light curves, because of its higher flexibility and easier application in current analysis programs compared to the Hadoop environment (Hahm et al. 2012).

The derived variability indices are made publicly available online. ${ }^{11}$ These variability indices can be used by others to try different machine learning algorithms without recalculating the indices. These publicly available variability indices can be also used to develop new variability indices that are better than, or complementary to, the indices adopted here.

\section{Clustering}

\subsection{Methods}

We apply three clustering methods to cluster light curves in the space of nine variability indices. The first method uses an infinite Gaussian mixture model (IGMM) with the Dirichlet process (DP; Ferguson 1973) as a prior on mixture mixing weights (Antoniak 1974; Robert 1996; Rasmussen 2000), and

\footnotetext{
9 http://research.cs.wisc.edu/htcondor/

$10 \mathrm{http}: / /$ hadoop.apache.org/

11 http://stardb.yonsei.ac.kr/
} 

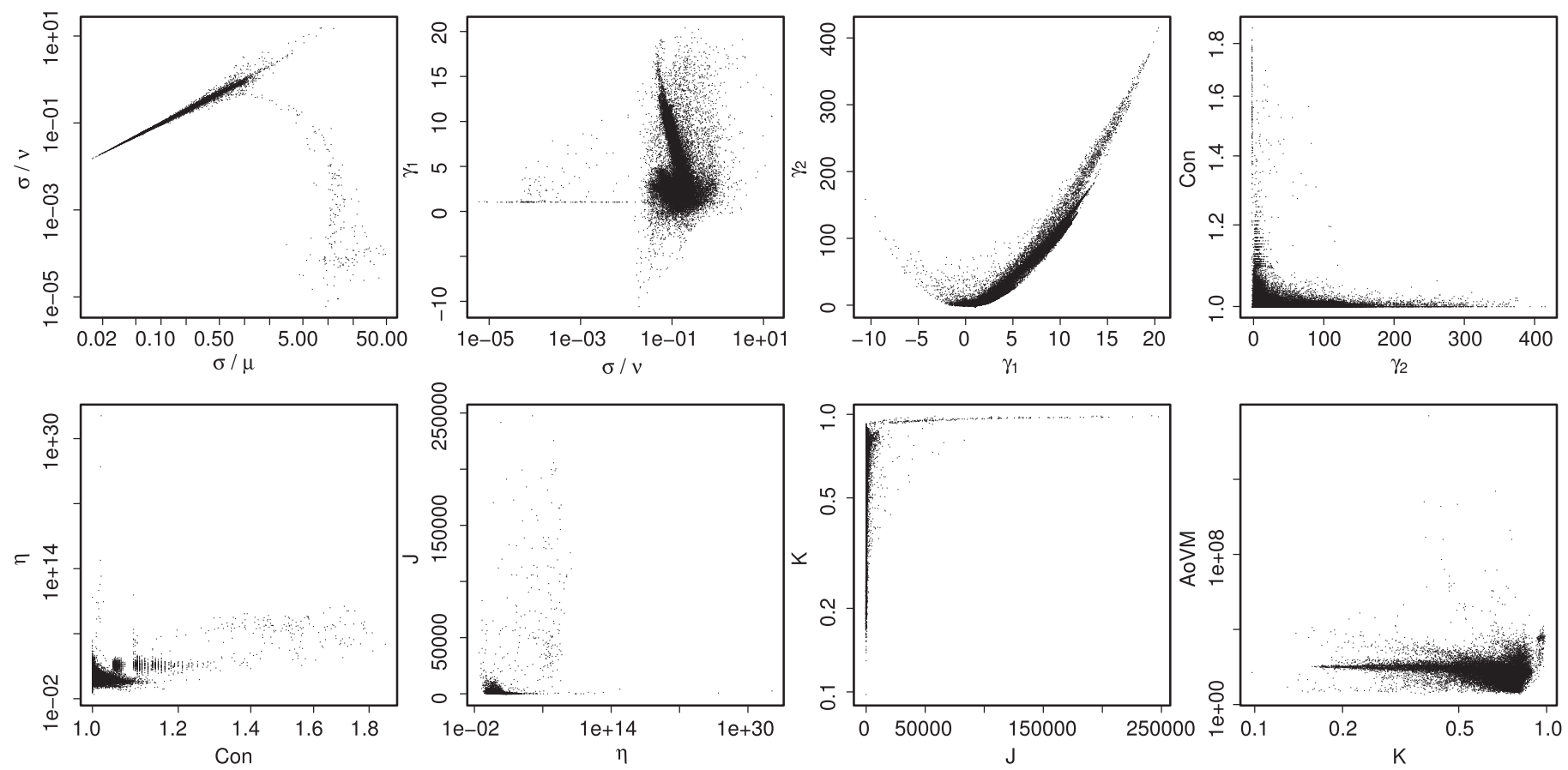

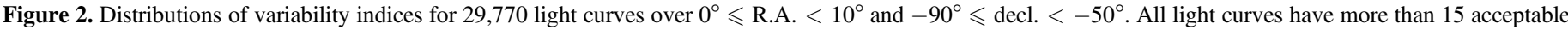
flux values. These distributions clearly show that the distribution of estimated flux values is not close to the normal distribution, which is commonly assumed.

this method has been used in our previous works (Shin et al. 2009, 2012). The second method, which is the focus of this paper, also uses the IGMM; however, this is done by exploiting the variational approximate Bayesian inference (Tzikas et al. 2008) rather than using MCMC (Markov Chain Monte Carlo) samples, which are used in the first method (Cao \& West 1996; Neal 2000; Blei \& Jordan 2006). The third method also uses the Gaussian mixture model, but it uses the expectation-maximization (EM) algorithm (Moon 1996) with finite Gaussian mixtures and information criteria to determine the best number of mixture components in the problem of model selection (Weakliem 1999; Zucchini 2000; Liddle 2007; Trotta 2008; Chaurasia \& Harel 2013). We present the mathematical foundations of these clustering algorithms in the Appendix. In the following, we summarize only the key properties of these methods, focusing on the known pros and cons.

All three clustering methods use Gaussian mixtures to describe the density distribution of a given data, i.e., variability indices of light curves, in the nine-dimensional space, as follows:

$$
\begin{aligned}
p(\boldsymbol{x})= & \sum_{c=1}^{C} \frac{w_{c}}{(2 \pi)^{D / 2}\left|\Sigma_{c}\right|^{1 / 2}} \\
& \times \exp \left[-\frac{1}{2}\left(\boldsymbol{x}-\boldsymbol{\mu}_{c}\right)^{T} \Sigma_{c}^{-1}\left(\boldsymbol{x}-\boldsymbol{\mu}_{c}\right)\right],
\end{aligned}
$$

where $c$ is an index that runs over $C$ mixture components, $\boldsymbol{x}$ is the vector of the variability indices, $D$ is the dimension of space (here $D=9$ ), and $w_{c}$ is a mixing fraction of each mixture component. Each mixture component is characterized by the vector of mean values (i.e., mixture centers) $\mu_{c}$ and the covariance matrix of the Gaussian distribution $\Sigma_{c}$ associated with the $c$ th mixture component.
The three clustering methods differ in the following two aspects. First, each method uses a different approach to find the best estimate of the parameters $w_{c}, \mu_{c}$, and $\Sigma_{c}$ or their posterior distributions, with assumptions regarding $C$ (i.e., the number of possible components) in the data. Second, cluster membership of light curves is determined differently using the estimated density distribution of the variability indices.

Both the first and the second methods using the IGMM with the DP are non-parametric Bayesian methods of density estimation and clustering. The key property of these methods is a way of considering an infinite number of mixture components by adding or removing components, relying upon the data and a prior distribution in the model. Adding more components can improve the quality of density estimation. However, this results in overfitting the model to the data. In the Bayesian approach, the problem of overfitting is controlled by the prior distribution of model parameters that regularize the model.

The difference between the first and second methods lies in how posterior distributions of parameters are estimated. The first method (hereafter referred to as IGMM-MCMC) uses the MCMC method to sample a posterior or a conditional posterior distribution, while the second method (hereafter referred to as IGMM-VB) uses the variational approximate Bayesian inference by deriving approximate posterior distributions. Because IGMM-VB is implemented as a deterministic optimization problem, IGMM-VB is generally much faster than the stochastic searching approach of IGMM-MCMC. Moreover, the MCMC approach requires a substantial storage space of sampling results and a tricky, insecure way of checking convergence in the MCMC samples (Marin \& Robert 2007). However, the posterior distribution derived in IGMM-VB is an approximate distribution, and the optimization solution can be a locally optimized description of the posterior distribution rather than a globally optimized solution. 
The third method (hereafter referred to as GMM-EM) is not Bayesian. Instead, it finds the best model parameters by using a maximum-likelihood approach with an iterative EM algorithm. Because GMM-EM requires knowing a predefined number of mixture components, GMM-EM finds the best estimation of the number of mixture components with the help of the modelselection criteria for a varying number of components.

In the step of determining the cluster membership, both IGMM-MCMC and IGMM-VB can simply adopt the maximum a posteriori (MAP) approach or a complicated assignment, using the derived posterior distribution of model parameters, while the GMM-EM method assigns light curves to a component with a maximal likelihood. When the cluster membership is described by a variable $Z$ for a light curve $\boldsymbol{x}$ with estimated model parameters, the GMM-EM method finds the mixture component $c$ that maximizes $p\left(Z=c \mid \boldsymbol{x} ; w_{c}, \boldsymbol{\mu}_{c}, \Sigma_{c}\right)$. On the other hand, we use the MAP assignment of $c$ in the IGMM-VB method, corresponding to the maximization of the approximated $\widetilde{p}(Z=c \mid \boldsymbol{x})$. In the IGMM-MCMC method, we determine cluster memberships, considering the distribution of $p(Z=c \mid x)$ derived from the MCMC samples and its uncertainty, instead of the MAP point estimation that we used in Papers I and II (see Lau \& Green 2007 for a discussion).

In short, the IGMM-MCMC method is the most flexible method among the three methods, while also being the slowest one. The GMM-EM method is the fastest, but the model is too simple, possibly obtaining an incorrect solution for the maximum likelihood and model selection. The IGMM-VB method is reasonably fast and flexible although it might not be as precise as the IGMM-MCMC method. Our choice of these three clustering models considers the possible scenario of clustering a large amount of data as quickly as possible by using the fast method first and then using not only the two slow methods later but also the ensemble method. The quick analysis of the new data in the fast clustering method will allow people to discover new variable candidates quickly while the slow and ensemble methods improve the reliability of the candidate detection later.

\subsection{Example Analysis}

In the detection of variable sources, our main concern is how the different clustering methods recover large mixture components that represent non-variable sources in a given data set. Therefore, we examine how well the fast methods find mixture distributions that can be consistent with the result of the slowest method, despite the differences in their algorithms.

To exemplify the clustering analysis, we analyze a single set, which consists of 10,026 light curves, using the three different clustering methods. Figure 3 shows the cumulative distribution of light curves in order of increasing cluster sizes, up to the top 10 largest clusters. The IGMM-MCMC method discovered 17 clusters, including eight clusters that have fewer than 30 member (i.e., the fraction $\sim 0.003$ ) light curves. Although IGMM-VB reveals 34 clusters, the number of member light curves in 15 clusters is fewer than 30. Meanwhile, we find 17 clusters in the result of the GMM-EM method, with only four clusters having fewer than 30 member light curves. In Figure 3, the different pattern obtained using the IGMM-VB method is mainly explained by the fact that IGMM-VB identifies more small clusters than the other methods. Considering the computation time, the IGMM-MCMC method is the slowest

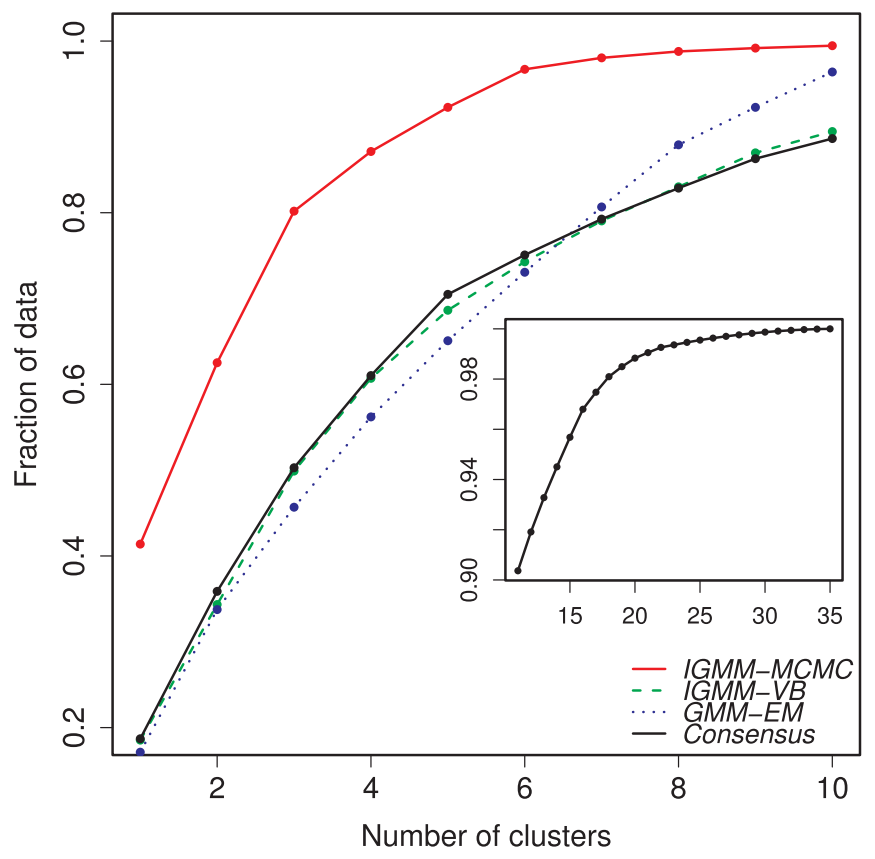

Figure 3. Cumulative fraction of light curves in the top 10 largest clusters. The different lines represent the distributions obtained by using the three different clustering methods, as well as the consensus clustering method. The inset shows the distribution derived in the consensus clustering for the rest of clusters, in addition to the top 10 largest clusters.

while the IGMM-VB and GMM-EM methods are about 64 and 10 times faster than the IGMM-MCMC method, respectively.

We find that all three clustering methods reveal similar structures of large clusters although the exact assignment of light curves into clusters is different. Figure 4 shows that the top three largest clusters found by the IGMM-MCMC method encompass about $80 \%$ of the data set, and these clusters capture a dominant structure in the projection onto the space of $K$ and AoVM. However, the results obtained using the IGMM-VB and GMM-EM methods exhibit differences in the fine details of the structures related to the top three largest clusters, as shown in Figure 5. This result implies that combining the results derived from multiple clustering methods can yield a consensus clustering solution for a given data set. In particular, clusters of non-variable sources (i.e., large clusters) can be defined more conservatively in the consensus clustering method compared to individual clustering methods.

To quantitatively measure the similarity between the results obtained using the three methods, we calculate three diagnostic quantities: $R, A R$, and $F$ (see Melnykov et al. 2012, for discussions). When $\mathrm{ID}_{1}$ and $\mathrm{ID}_{2}$ are clustering memberships derived from two methods for $N$ light curves, the Rand index $R$ is defined as follows (Rand 1971):

$$
R\left(\mathrm{ID}_{1}, \mathrm{ID}_{2}\right)=\left(N_{\mathrm{sc}}+N_{\mathrm{dc}}\right) /{ }_{N} C_{2},
$$

where $N_{\mathrm{sc}}$ is the number of pairs of light curves that are found in the same cluster in both $\mathrm{ID}_{1}$ and $\mathrm{ID}_{2}$ among ${ }_{N} C_{2}$ possible pairs, and $N_{\mathrm{dc}}$ represents the number of pairs that are in different clusters of $\mathrm{ID}_{1}$ and $\mathrm{ID}_{2}$. Therefore, $N_{\mathrm{sc}}+N_{\mathrm{dc}}$ measures pairwise similarities between two clustering results. However, $R$ does not consider the number of disagreeing pairs, which correspond to pairs found in the same cluster using only one method. A completely consistent clustering result corresponds to $R=1$. Following the principle of $R$, the definition of the adjusted Rand 

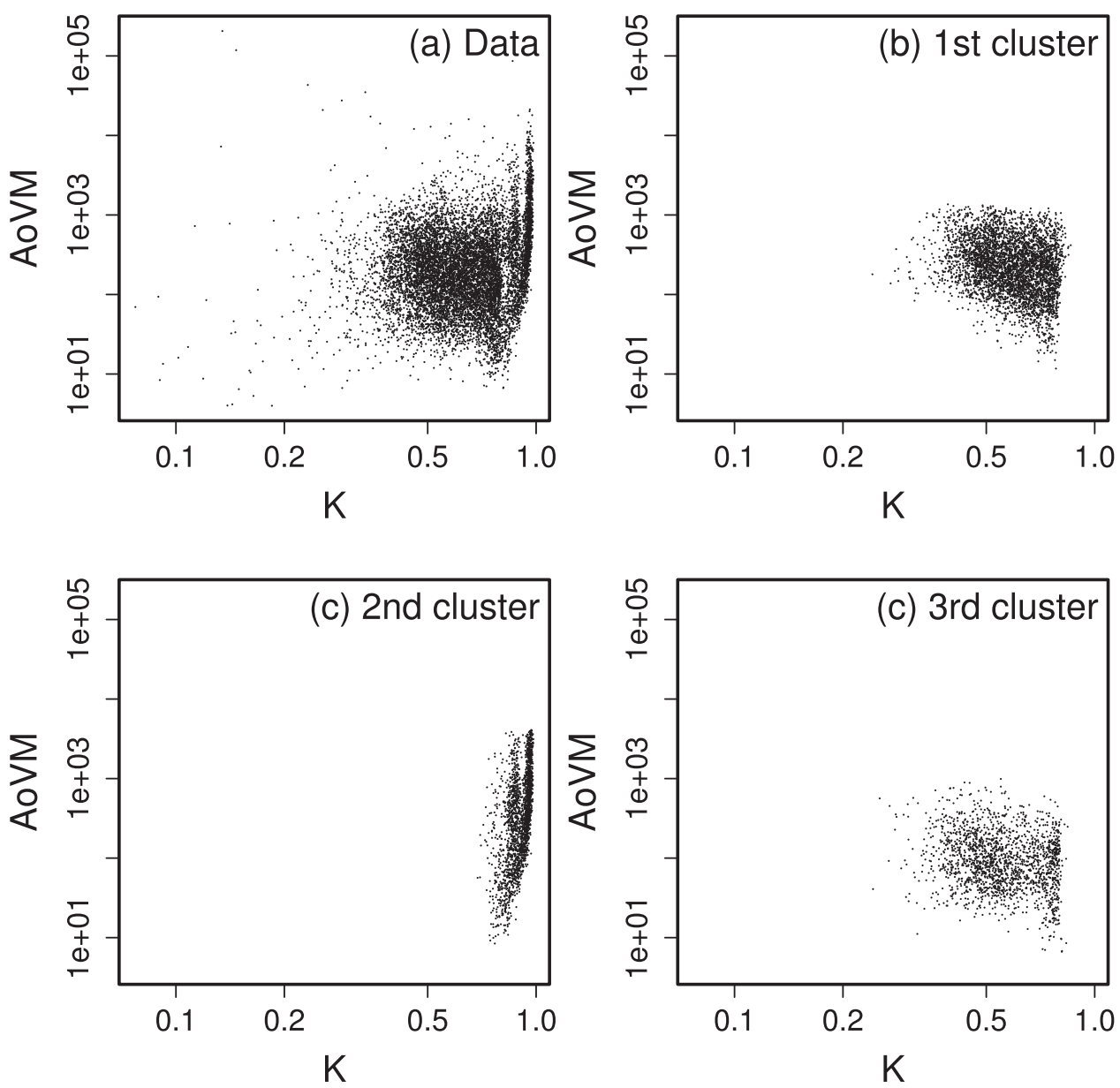

Figure 4. Distributions of $K$ and AoVM in the example data and the results obtained using the IGMM-MCMC method. (a) The entire example data set. (b) The first largest cluster. (c) The second largest cluster. (d) The third largest cluster.

index $A R$ (Hubert \& Arabie 1985) is

$$
A R\left(\mathrm{ID}_{1}, \mathrm{ID}_{2}\right)=\frac{R\left(\mathrm{ID}_{1}, \mathrm{ID}_{2}\right)-E\left[R\left(\mathrm{ID}_{1}, \mathrm{ID}_{2}\right)\right]}{1-E\left[R\left(\mathrm{ID}_{1}, \mathrm{ID}_{2}\right)\right]},
$$

where $E\left[R\left(\mathrm{ID}_{1}, \mathrm{ID}_{2}\right)\right]$ is the expected value of $R$ with pairs coincidentally counted in $\mathrm{ID}_{1}$ and $\mathrm{ID}_{2}$. Accordingly, $A R$ is equal to $R$ minus the $R$ expected at random after being normalized by the possible maximum value of $R$ minus the $R$ expected at random. $F$ considers the differences of the number of clusters in $\mathrm{ID}_{1}$ and $\mathrm{ID}_{2}$ (Fowlkes \& Mallows 1983) as described by the following definition:

$$
F\left(\mathrm{ID}_{1}, \mathrm{ID}_{2}\right)=\frac{2 N_{\mathrm{sc}}}{\sqrt{\sum_{c=1}^{C_{1}} n_{c}\left(n_{c}-1\right) \cdot \sum_{c=1}^{C_{2}} n_{c}\left(n_{c}-1\right)}},
$$

where $n_{c}$ is the size of the $c$ th cluster when the numbers of clusters found in $\mathrm{ID}_{1}$ and $\mathrm{ID}_{2}$ are $C_{1}$ and $C_{2}$, respectively.

Table 2 lists the measured $R, A R$, and $F$ for the clustering results derived from the three methods. All three quantities attain the value of 1 only if two methods produce an exactly identical clustering result. All three measurements prove that the IGMM-VB and GMM-EM methods are the most similar among the three possible pairs of methods, regardless of the similarity measurements used. We also find that more than $80 \%$ of the light curve pairs are consistently clustered using the three different methods as shown for $R$.

\subsection{Consensus Clustering}

We combine the results from the multiple different clustering methods by using the method of "the best one element moves" with one of the three clustering results as a seed clustering result (see Goder \& Filkov 2008 for details). Among several possible ways of consensus clustering, we adopt a simple fast approach. In short, one of the three clustering results is chosen as a candidate for the solution of consensus clustering if the candidate has the smallest averaged discrepancy, which is measured by the Rand index (i.e., Equation (2)), with two other clustering results. Therefore, finding the best consensus clustering result becomes an optimization problem of finding a solution sharing the highest similarity with all three preexisting clustering results. Starting from this candidate solution, the method of "the best one element moves" changes the clustering membership of one element in the candidate clustering solution until the averaged discrepancy of the solution with respect to the three clustering results decreases (e.g., Filkov \& Skiena 2003; Zheng et al. 2011).

As presented in Table 2, the clustering result of the IGMMVB method becomes a promising candidate solution of the best consensus clustering, and then the consensus clustering result resembles the result obtained using the IGMM-VB method with small changes guided by differences in the results from other two clustering methods, as shown in Figure 3. The consensus clustering result has the top 10 largest clusters containing about $89 \%$ of the example light curves. About $98 \%$ 

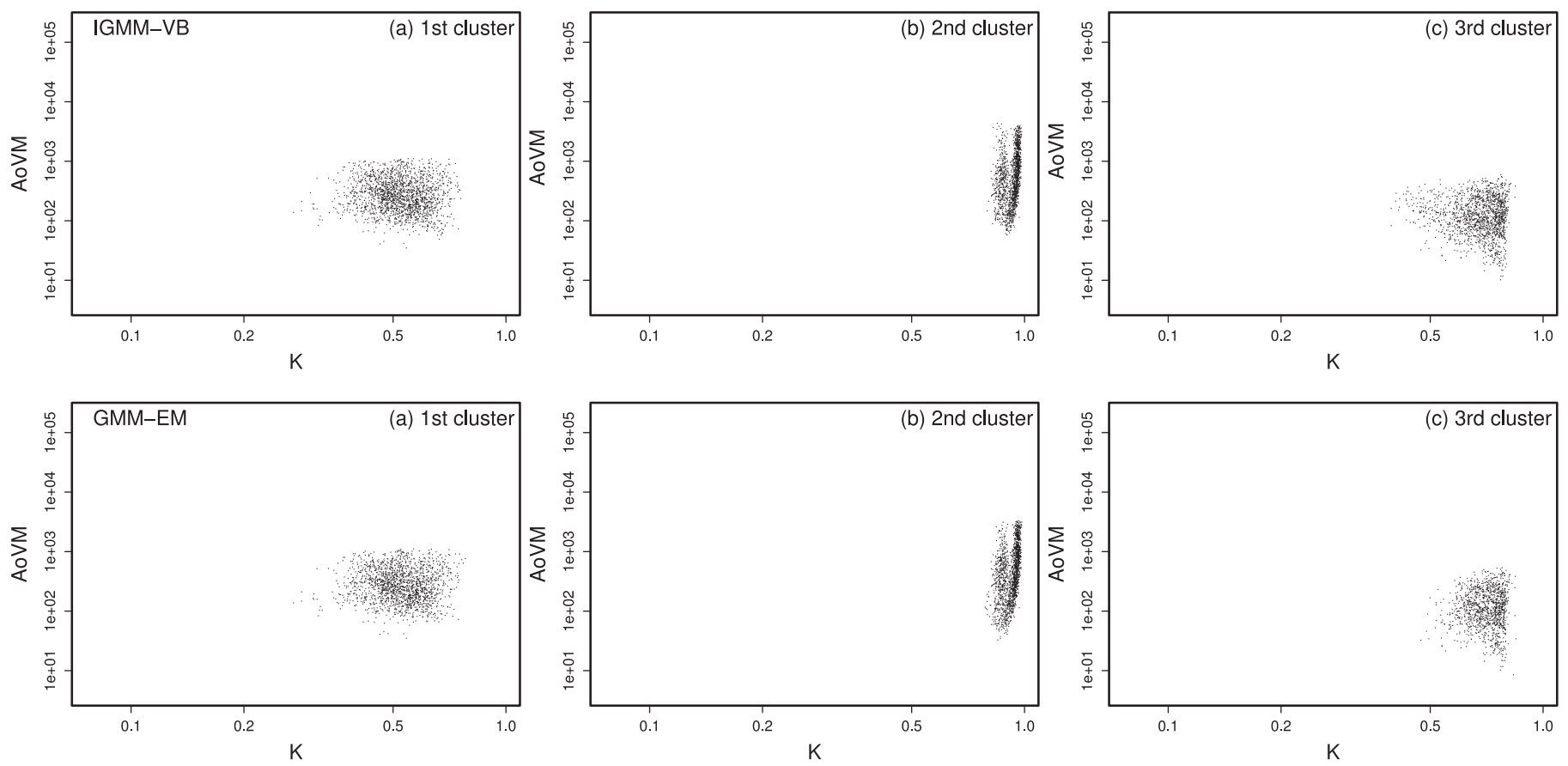

Figure 5. Distributions of $K$ and AoVM in the top three largest clusters found in the IGMM-VB (top) and GMM-EM (bottom) results. From left to right, each column corresponds to the first, second, and third largest clusters, respectively.

Table 2

Similarity of Clustering Results

\begin{tabular}{lccc}
\hline \hline Two Methods & $R$ & $A R$ & $F$ \\
\hline IGMM-MCMC, IGMM-VB & 0.821 & 0.424 & 0.559 \\
IGMM-MCMC, GMM-EM & 0.815 & 0.406 & 0.542 \\
IGMM-VB, GMM-EM & 0.938 & 0.689 & 0.724 \\
\hline
\end{tabular}

of the data are recovered in the top 18 largest clusters. Therefore, many small clusters exist in the consensus clustering result compared to the IGMM-MCMC method.

The consensus clustering result implies that many real variable candidates might be mixed together with non-variable light curves, conservatively defining the boundary between variable and non-variables objects. In order to measure this change quantitatively, we estimate two quantities: the DaviesBouldin (DB) index (Davies \& Bouldin 1979) and the Dunn index (Dunn 1973), which are commonly used as diagnostic measurements of the clustering quality or patterns (e.g., Maulik \& Bandyopadhyay 2002; Vendramin et al. 2010). The DB index, which was used in Paper I (Shin et al. 2009), measures the ratio of the dispersion of cluster members to the separations between clusters by using the following definition:

$$
\mathrm{DB}=\frac{1}{N_{C}} \sum_{i=1}^{N_{C}} \max _{j=1, \ldots, N_{C}, i \neq j}\left(\frac{s_{i}+s_{j}}{d_{i j}}\right),
$$

where $s_{i}$ is the average distance of members with respect to the cluster center for the cluster $i$ and $d_{i j}$ is the distance between the centers of two clusters $i$ and $j$ for the number of clusters $N_{C}$. The definition of the Dunn index is

$$
\text { Dunn }=\min _{i=1, \ldots, N_{C}}\left\{\min _{j=1, \ldots, N_{C}, i \neq j}\left(\frac{\delta_{i j}}{\max _{k=1, \ldots, N_{C} \Delta_{k}}}\right)\right\},
$$

Table 3

Estimation of the DB and Dunn Indices

\begin{tabular}{lcccc}
\hline \hline Index & IGMM-MCMC & IGMM-VB & GMM-EM & Consensus \\
\hline DB & 2.58 & 13.8 & 5.02 & 11.3 \\
Dunn & $6.09 \times 10^{-7}$ & $1.05 \times 10^{-6}$ & $1.66 \times 10^{-6}$ & $3.88 \times 10^{-7}$ \\
\hline
\end{tabular}

where $\delta_{i j}$ is the minimum distance of members between two clusters $i$ and $j$, and $\Delta_{k}$ is the maximum distance between two members in the cluster $k$ with $N_{C}$ clusters. We use the $L^{2}$ norm as the distance when measuring both indices. While small values of the DB index imply well-separated compact clusters, a large Dunn index represents a distribution of compact clusters with a large separation among them.

Table 3 shows that the consensus clustering result yields less compact and less separated clusters compared to the results from the IGMM-MCMC and GMM-EM methods, considering the DB index estimation. However, the Dunn index suggests that the consensus clustering result can be better than that obtained using the IGMM-MCMC method. This mixed result of the comparison between the IGMM-MCMC method and consensus clustering can be understood by noting that the Dunn index, which sensitively depends on maximum and minimum distances, can be easily changed by the distribution of small, largely separated clusters. As shown in Figure 3, several small clusters in the consensus clustering result are defined by separating them out from large clusters found in the IGMM-MCMC result. Therefore, although the DB index dominated by the structure of large clusters demonstrates better clustering in the IGMM-MCMC result than in the consensus clustering result, the Dunn index is higher in the consensus clustering result than in the IGMM-MCMC clustering result. 


\section{Selecting Variable Candidates}

We propose two ways in which the clustering results can be used to find variable candidates as we did in Paper I. Since nonvariable sources are expected to be found in large clusters, first, we can select light curves in small clusters as possible candidates of variable sources. Second, if the largest cluster describes well the distribution of non-variable sources in the multidimensional space of the variability indices, we can use a distance metric to identify as variable candidates light curves that are distant from the largest cluster in the multidimensional space. In Paper I, we adopted the Mahalanobis distance $\left(D_{M}\right)$ (Bishop 2007) as a distance metric with a covariance matrix of the largest cluster (see Jogesh Babu et al. 2008 for astronomical examples). Because even small clusters can be close to the most dominant cluster in the multidimensional feature space, a conservative approach can adopt both the selection using cluster sizes and that using cluster distances from the largest cluster.

In reality, the way to select interesting and reliable variable candidates is more complicated than what we propose here with the clustering results. Depending on the research interests and extra information on sources, selection methods can be polished to reflect practical constraints. In particular, users of our analysis results should know that the clustering analysis is still affected by the intrinsic limitation of the WASP observation such as spatial resolution. For example, the existence of infrared detection can be a required condition to refine the candidate selection procedure proposed here. Moreover, the poor spatial resolution of data may make it extremely difficult to separate out reliable stellar objects from extended sources with variability patterns in their light curves. In this case, the identification of variable candidates as point sources in other observations will be required in addition to detecting variable light curves in data with poor spatial resolution. Therefore, the proposed ways to discover variable candidates should be considered as a minimal guide for people who would like to use the results of our analysis.

\subsection{Light Curves in Minor Clusters}

We compare the variable candidates selected in the results of the IGMM-VB method with those obtained by the consensus clustering method. We provide the clustering results from the IGMM-VB method online for public use. Because the IGMMVB method is the flexible Bayesian model compared to the GMM-EM method, and it is computationally much cheaper than the IGMM-MCMC method, we use the results obtained using this method as a main way to discover variable candidates.

In the clustering results of the example data presented in the previous sections, the number of variable candidates is 317 and 321 in the IGMM-VB and consensus clustering results, respectively, when we select light curves found in clusters that comprise less than $1 \%$ of the data. This cut is arbitrarily chosen depending on how strongly it is desired to suppress the contamination of non-variable light curves among the candidates. The number of these small clusters is 19 in both the IGMM-VB and consensus clustering results. Among these candidates, all 317 light curves selected using the IGMM-VB method are chosen as variable candidates by the selection using the consensus clustering result.
Figure 6 shows examples of variable and non-variable light curves selected in the IGMM-VB clustering results. Although the light curves of both variable and non-variable candidates look similar, their distributions in the multidimensional space of the variability indices separate out these light curves into multiple clusters. These examples also show that a simple estimation of flux variation such as dispersion is not sufficiently powerful enough to separate out variable candidates from nonvariable objects, as implied by the comparison between 1SWASP J231041.63+402849.9 and 1SWASP J221311.94 +483003.8 . The light curves shown here still exhibit typical systematic trends of the original data before the detrending correction (Butters et al. 2010) is applied. Therefore, it is not easy to catch recognizable features of variability even in the light curves of variable candidates, implying that visual detection of variability signals might be challenging.

The examples of the variable candidates presented in Figure 6 exhibit uncommon values of some variability indices compared to others being selected as variable candidates. The values of AoVM for the variable candidates are about 15,965, 205,374, and 86,233 for 1SWASP J231443.22+371307.7, 1SWASP J225515.55+475810.3, and 1SWASP J231041.63 +402849.9 , respectively. The range of AoVM is much narrower in the non-variable light curves such that the values for 1SWASP J221217.27+485719.7, 1SWASP J221209.83 +484624.8 , and 1SWASP J221311.94+483003.8 are about 44,52 , and 476 , respectively. In the case of the variable candidate 1SWASP J231041.63+402849.9, $J \sim 1,146,160$ is also much larger than the values found for others. The values of $J$ are between 186 and 28,375 for the non-variable objects shown in Figure 6.

Figure 7 shows example light curves of variable candidates that are not selected by the IGMM-VB method but are selected by the consensus clustering method. We do not detect any visually distinctive differences between the candidates found by the IGMM-VB method and those found by the consensus clustering method, implying again that visual perception of raw light curves is not suitable for identifying variability signals in data such as the WASP data. Inspecting the variability indices of these light curves provides a hint to the reason why they are weakly separated from the dominant population of non-variable light curves. For example, 1SWASP J223910.69+444734.4, 1SWASP J224638.55+460649.0, and 1SWASP J225111.89 +475100.7 have $\gamma_{1} \sim 532,523$, and 644 , respectively. These values are higher than the ones we find for other light curves, as shown in Figures 2. The values of $\gamma_{2}$ are also higher than others for these light curves. However, the large values attained by only these two indices do not result in a large separation of these light curves from the majority of variability indices. In the case of 1SWASP J231018.73+424237.6, all variability indices deviate marginally from the major concentration of the indices shown in Figure 2.

We examine whether any known variable stars are included in the examined data. Matching the data to the VSX catalog (Watson 2006) with the SuperWASP coordinates and a 6 arcsec search radius reveals one known variable star for 1SWASP J221937.28+441703.2. However, when increasing a search radius, we find two more objects, 1SWASP J225427.54 +482557.8 and 1SWASP J230311.84+423909.2, in the VSX catalog. Searching the SIMBAD database (Wenger et al. 2000) with a 6 arcsec search radius recovers 1SWASP J221937.28 $+441703.2, \quad$ 1SWASP J225427.54+482557.8, 1 SWASP 

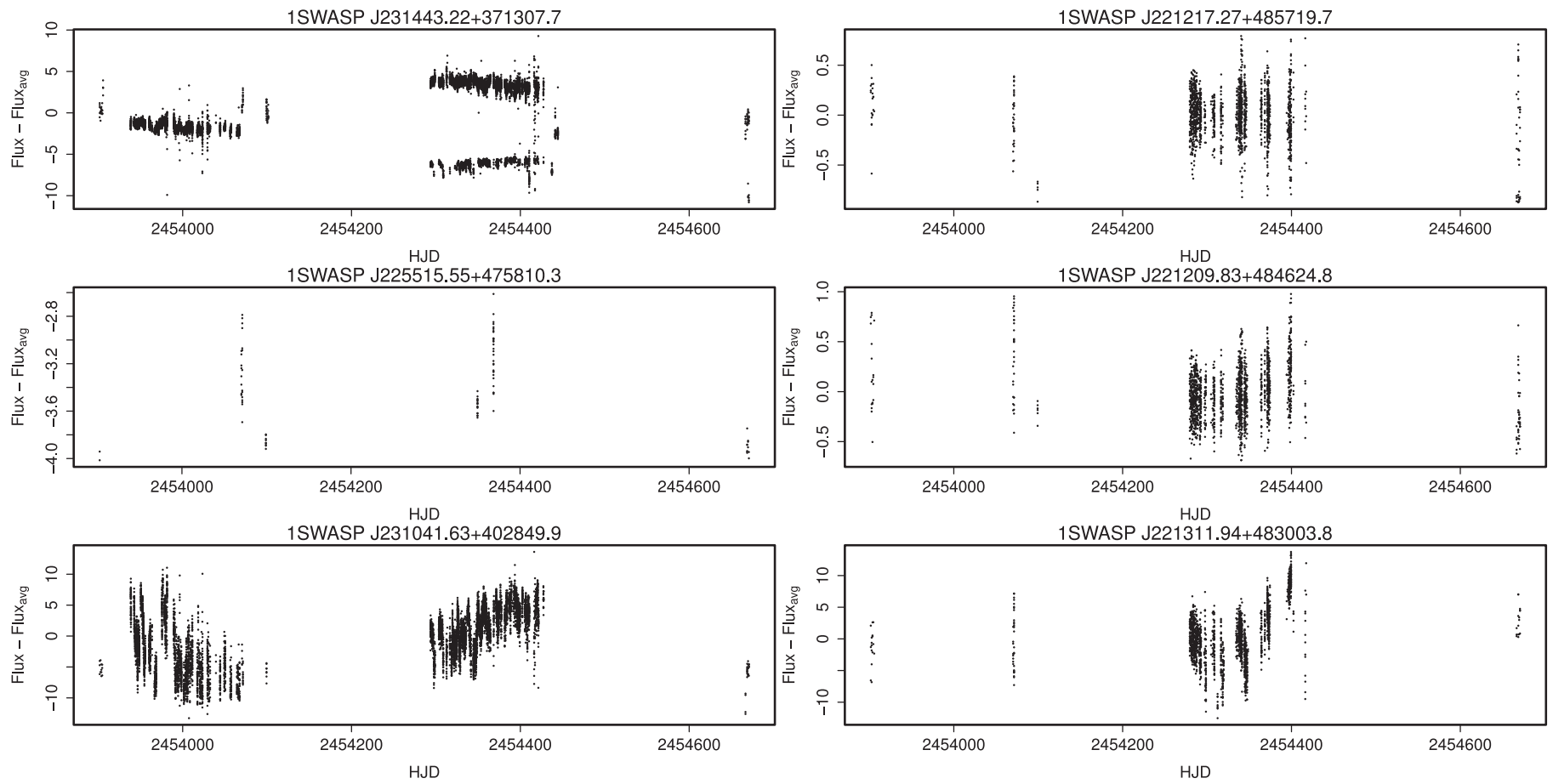

Figure 6. Example light curves of variable candidates (left) and non-variable candidates (right) selected by the IGMM-VB method. We consider as variable candidates the objects found in clusters that include less than $1 \%$ of the data. The names in each panel represent the equatorial coordinates assigned by the first data release of the

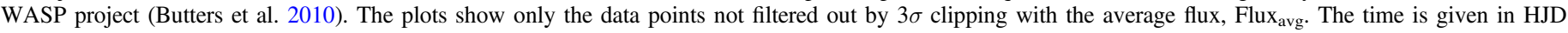
(Heliocentric Julian Day). The unit of flux is micro-Vega in all plots hereafter.

J230311.84+423909.2, and 1SWASP J231234.00+364120.9 as $\mathrm{V}^{*}$ BS Lac, $\mathrm{V}^{*}$ FT Lac, GSC 03224-02811, and BD+35 4979 , respectively. It is not obvious that their light curves, shown in Figure 8, have visually detectable well-defined variability signatures. None of these objects compose the small clusters in either the IGMM-VB or consensus clustering results. Although these objects are included as eclipsing variable stars in the SIMBAD database, the nature of their variability is not confirmed in their WASP light curves without any previous reports analyzing the WASP light curves.

1SWASP J221937.28+441703.2 (i.e., $\mathrm{V}^{*}$ BS Lac) and 1SWASP J230311.84+423909.2 (i.e., GSC 03224-02811) help us understand how known variable objects can be hidden in clusters identified using our clustering methods. Both objects are included in the VSX catalog as eclipsing variables, with suggested periods of 2.8142 and 0.4999 days, respectively. There is no known previous analysis of their SuperWASP light curves in any other literature. Figure 9 shows their light curves in phase space, for the suggested periods. Although the raw light curve of $\mathrm{V}^{*}$ BS Lac demonstrates obvious features of eclipsing variables, the systematic effects embedded in the data points significantly offset the magnitude of the phased light curve. The light curve corrected for systematic effects by the SuperWASP team (Collier Cameron et al. 2006) improves the quality of the light curves in the phase space even though the correction is not perfect. Importantly, $\mathrm{V}^{*} \mathrm{BS}$ Lac is included in the fourth largest cluster in the results obtained by both the IGMM-VB and consensus clustering methods (see Figure 3). Therefore, this object can still be considered as a possible variable candidate with a low-level weak variability signal over systematic and random errors. In the case of GSC 03224-02811, the variability signal is not apparent in the SuperWASP light curve as shown in Figure 9. This object is included in the fourth largest cluster found in both the IGMMVB and consensus clustering results.

\subsection{Light Curves with a Large $D_{M}$ from the Largest Cluster}

When using the $D_{M}$ from the largest cluster as an indicator of the extent to which light curves differ from dominant ordinary light curves (i.e., non-variable light curves), we can select variable candidates by measuring their $D_{M}$. Although this can be done in principle, this distance measurement from the single largest cluster might not be effective when the fraction of the data in the largest cluster is not strongly dominant. For example, some light curves can have a large $D_{M}$ from the first largest cluster even though they can be close to the third largest cluster.

Figure 10 shows the distribution of $D_{M}$ measured with respect to the largest cluster in the IGMM-VB and consensus clustering results. The centers of the largest clusters are similar in the IGMM-VB and consensus clustering results. Their covariances are not significantly different so that the maximum $D_{M}$ found by the consensus clustering method is about 4730 , which is close to the maximum $D_{M} \sim 5062$ found by the IGMM-VB method. The broad distribution of $D_{M}$ for the large clusters (i.e., non-variable candidates) also implies that the quality of the raw photometric time-series data in the SuperWASP data might not be satisfactory for detecting weak variability in light curves with low signal-to-noise ratios (see Section 3.3 in Paper I).

The $99.9 \%$ cut of the $D_{M}$ for the largest cluster turns out to be an ineffective measure to isolate variable candidates from others. As shown in Figure 10, the fraction of light curves in the largest cluster is not high enough to make the $99.9 \%$ cut enclose most non-variable candidates inside the cut. All variable candidates found using the method explained in 
1SWASP J223910.69+444734.4

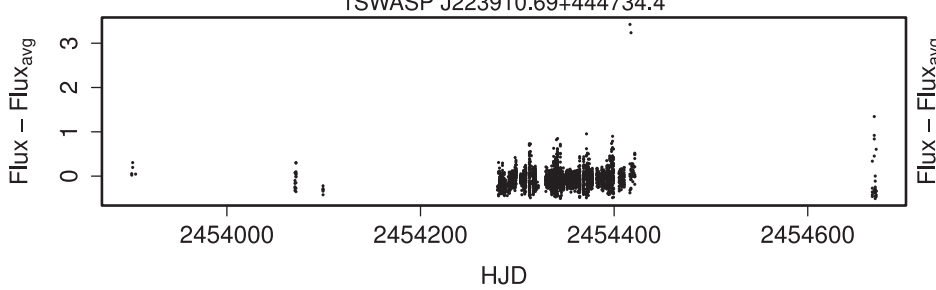

1SWASP J225111.89+475100.7

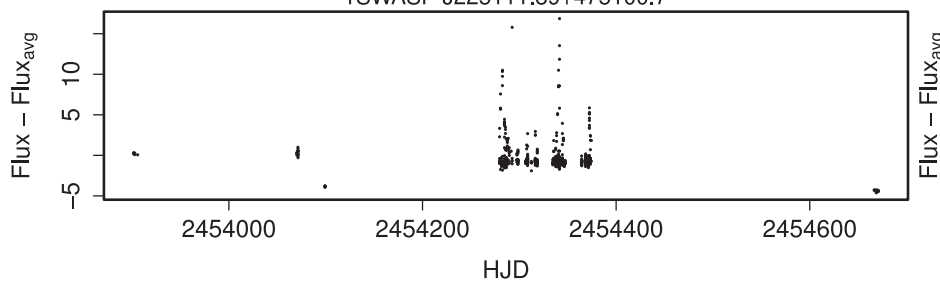

1SWASP J224638.55+460649.0

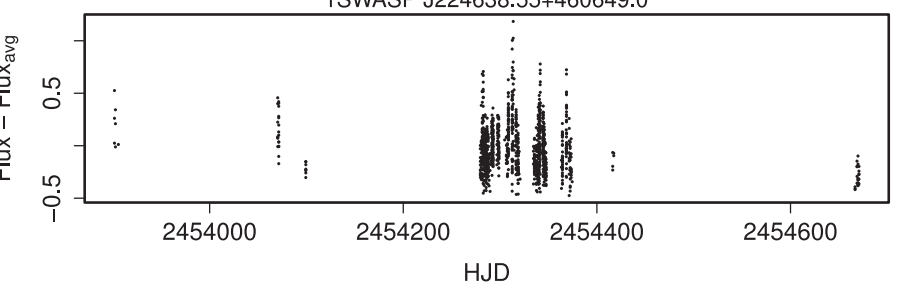

1SWASP J231018.73+424237.6

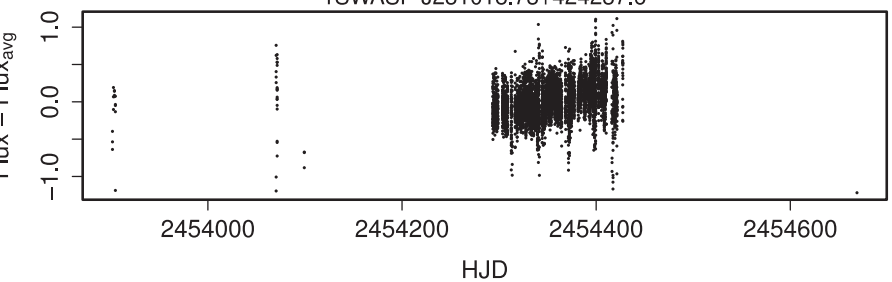

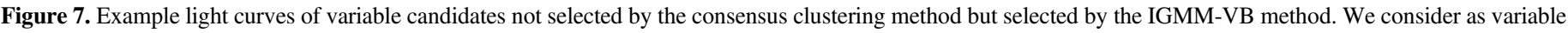
candidates the objects found in clusters that comprise less than $1 \%$ of the data. Other properties of the plots are the same as those of Figure 6.

Section 4.1 have much higher $D_{M}$ than the $99.9 \%$ cut (see the inset plots in Figure 10). In general, objects with very high $D_{M}$ are found only in minor clusters.

The values of $D_{M}$ for the two known variables 1SWASP J221937.28+441703.2 (i.e., $\mathrm{V}^{*}$ BS Lac) and 1SWASP J230311.84+423909.2 (i.e., GSC 03224-02811) also demonstrate how weakly these objects are separated from the largest cluster in the multidimensional space of the variability indices. In the results produced by the IGMM-VB method, $\mathrm{V}^{*}$ BS Lac and GSC 03224-02811 have $D_{M} \sim 29.33$ and 11.88, respectively, demonstrating that the variability signal of GSC 03224-02811 is much weaker than that of $\mathrm{V}^{*}$ BS Lac in the SuperWASP light curves as shown in Figure 9. The values of $D_{M}$ are 29.01 and 11.73 for $\mathrm{V}^{*}$ BS Lac and GSC 03224 -02811 , respectively, in the consensus clustering result, indicating that these objects are not very far from the largest cluster as shown in Figure 10.

As we proposed in Papers I and II, it is possible to combine the two selection criteria of the cluster size and the $D_{M}$ from the largest cluster, although this way cannot be effectively applied to the data presented in Figure 10. If the given data of the light curves are described in terms of a few dominant clusters, we can productively select variable candidates by excluding the large cluster members and light curves within the significance $D_{M}$ cut from the single largest cluster or multiple large clusters.

\section{Variable Candidates Found in Other Catalogs}

We match various large catalogs to the SuperWASP coordinates of all light curves analyzed here for a fixed 6" search radius in order to narrow down to interesting variable candidates easily and to figure out basic properties of the candidate objects such as their stellar spectral types. In particular, probing multiwavelength colors allows us to identify reliable stellar spectral types (e.g., Davenport et al. 2014) and to discover unusual objects such as objects with mixed stellar colors (e.g., Krisciunas et al. 1998; Verbeek et al. 2014). Using other optical or near-IR data such as the Sloan Digital Sky Survey (SDSS) and 2MASS data is also important to check whether any objects are selected as variable candidates because of the intrinsic limitations of wide-field small-aperture telescopes such as poor spatial resolution, which makes reliable separation of point sources from extended sources difficult.
When selecting variable candidates, light curves of extended sources should be avoided because of the systematically different properties of their photometric measurements. Classification provided by the SIMBAD astronomical database (Wenger et al. 2000) helps us find extended sources in variable candidates found by either method suggested in Sections 4.1 or 4.2. We find that variable candidates only selected by the $99 \% D_{M}$ cut include some known galaxies. As the examples in Figure 11 show, the light curves of these galaxies are not so apparently different from those shown in Figures 6-8. As expected for the poor spatial resolution of the SuperWASP data, 2MFGC 17130 corresponds to three SuperWASP light curves: 1SWASP J224632.55+475613.0, 1SWASP J224632.62+475612.7, and 1SWASP J224632.84+475610.6. 2MASX J22384761+4744336 also coincides with three SuperWASP light curves: 1SWASP J223847.41+474431.3, 1SWASP J223847.44+474433.2, and J223847.47+474432.2.

In the infrared range of wavelengths, we search for objects corresponding to the SuperWASP objects in the IRAS point source catalog (Helou \& Walker 1988), IRAS faint-source catalog (Moshir et al. 1990), AKARI/IRC All-sky Point Source Catalog (version 1.0; Ishihara et al. 2010a, 2010b), AKARI/FIS All-sky Survey Bright Source Catalog (version 1.0; Yamamura et al. 2010), and AllWISE source catalog from WISE observations (Wright et al. 2010).

In the analysis of 10,026 example light curves, a large number of variable candidates found in Section 4.1 has corresponding WISE objects. Figure 12 shows the WISE colors of the example light curves and variable candidates selected as minor clusters comprising less than $1 \%$ of the data in the IGMM-VB clustering result. We find only one more additional WISE object in the variable candidates selected by the consensus clustering method compared to the IGMM-VB method. The general distribution of colors is consistent with the overall distribution for stars (e.g., Wright et al. 2010; Jarrett et al. 2011; Nikutta et al. 2014). Although most objects, including variable candidates, have colors around 0 , which represent typical stellar objects, some objects have dust-obscured stellar colors or extragalactic sources' colors, which are much redder than others in W2 - W3 (Jarrett et al. 2011; Hainline et al. 2014; Nikutta et al. 2014; Theissen \& West 2014). 

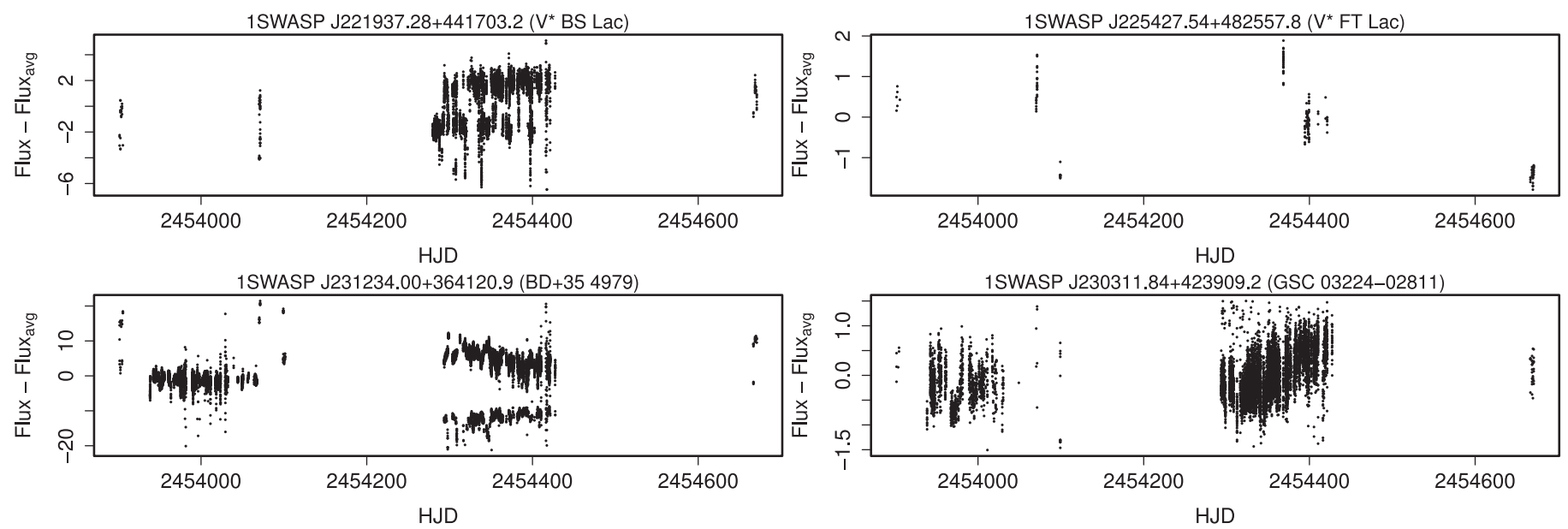

Figure 8. Example light curves of non-variable objects with SIMBAD classification as variable stars. These objects are found to be located in large clusters that represent non-variable objects. Other properties of the plots are the same as those of Figure 6, except for the SIMBAD object identifications.

Figure 13 shows example light curves of the variable candidates found with SIMBAD designations and WISE colors in the IGMM-VB clustering result. The variable candidates found as minor clusters using the IGMM-VB method include $\mathrm{BD}+434223$ (WISEA J223232.08+442717.5) and TYC 2763-544-1 (WISEA J231443.23+371305.0), TYC 32161143-1 (WISEA J230737.25+391331.9), and 2MASS J22510107+4952524 (WISEA J225101.07+495252.4). None of these examples demonstrate a strong variability signal in the WISE observation, considering the ALLWISE variability flag. However, 2MASS J22510107+4952524, which has a significantly red color, might be an active galactic nucleus (Edelson \& Malkan 2012), which is generally a variable source. This identification also implies that photometry of this possibly extended object might not be sufficiently precise to recover the full amplitude of the corresponding variability signal. Comparing these examples to the cases presented in Figures $6-8$, we again find that visual recognition of variability signals in raw light curves is not straightforward.

Although SDSS Data Release 10 (DR 10; Ahn et al. 2014) does not cover the example light curves, cross-matching the light curves to the 2MASS All-sky Catalog of Point Sources (Skrutskie et al. 2006) allows us to probe the properties of most of the variable candidates, resulting in 9,967 2MASS objects corresponding to 10,026 example light curves. Moreover, the 2MASS colors can be used to deduce optical SDSS colors such as $(g-i)$ if extinction is not a problem (e.g., Davenport et al. 2014). The distribution of the 2MASS colors in Figure 14 indicates that most variable candidates follow a well-defined stellar locus. The 2MASS colors $\left(J-H, H-K_{s}\right)$ of the examples presented in Figure 13 are $(0.811,0.244),(0.300$, $0.124),(0.224,0.094)$, and $(0.920,0.685)$ for BD+43 4223, TYC 2763-544-1, TYC 3216-1143-1, and 2MASS J22510107 +4952524 , respectively. Suspected to be a possible non-stellar object in the WISE color distribution (Edelson \& Malkan 2012), the color of 2MASS J22510107+4952524 does not follow the typical stellar locus in 2MASS colors, too. Although $J-K_{s}$ can be used to infer the spectral types and variability types of variable candidates (e.g., Borissova et al. 2009; Cioni et al. 2014), the photometric estimation of spectral types should be used with caution because of the Galactic reddening as presented in Figure 14.
As a main source of all-sky ultraviolet observation, we use the GALEX GR6/7 Data Release and its pipeline-processed photometric catalog. ${ }^{12}$ The GALEX ultraviolet color FUV NUV is a useful tool for investigating Galactic hot stellar objects (e.g., Bianchi et al. 2007; Pradhan et al. 2014) although dust extinction affects the GALEX bands more severely compared to the optical or near-infrared bands. 2MASS J23181552+4132077 (i.e., 1SWASP J231815.51+413207.7) with $\left(J-K_{s}, \mathrm{FUV}-\mathrm{NUV}\right)=(0.20 \pm 0.03,4.61 \pm 0.36)$ is the only object that is a variable candidate in the IGMM-VB clustering result as described in Section 4.1, appearing as a good photometric object in both 2MASS and GALEX photometric catalogs. The colors of this variable candidate conform to the expected temperature ranges of the instability strip if extinction does not substantially change the apparent color (see Figure 15). The cluster including this variable candidate comprises only $0.3 \%$ of the total number of objects in the sample, being the 20th largest cluster.

\section{Online Database}

In the online database, ${ }^{13}$ we provide the variability indices of all objects as well as the IGMM-VB clustering results. If database users would desire to analyze the data using various machine learning or statistical methods of their choice, they can acquire the variability indices in the database as either bulk files or query results. Moreover, our online database allows other researchers to combine our clustering results with clustering outcomes obtained by analyzing other data such as the colors of objects or light curves acquired in other observations. In particular, users can search both our NSVS (Shin et al. 2009, 2012) and SuperWASP variability analysis results in the single database. Because the spatial and magnitude ranges of both data sets overlap significantly, we expect that users will be able to attempt detecting long-term variability by checking both data sets. The online database is also accessible through a simple cone search of the Virtual Observatory interface (Williams et al. 2008).

The database also serves as an interactive interface with downloadable files cross-matching the analyzed SuperWASP light curves to other catalogs and databases: SIMBAD (Wenger

\footnotetext{
12 http://galex.stsci.edu/

13 http://stardb.yonsei.ac.kr/
} 

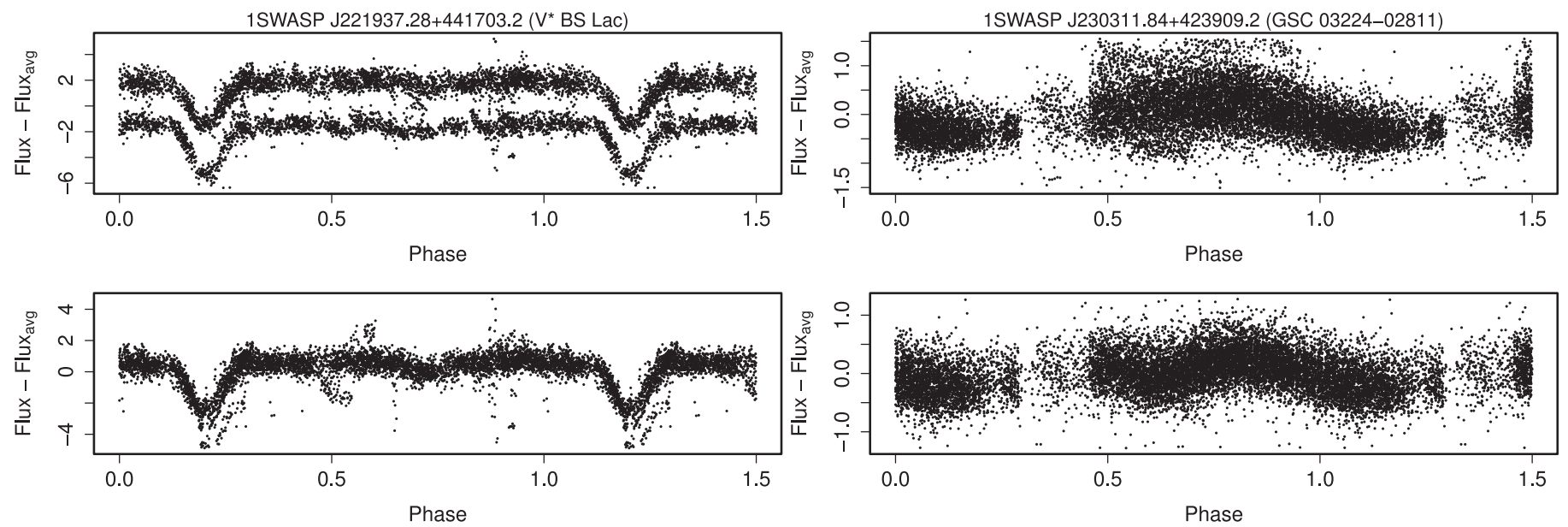

Figure 9. Light curves of two known eclipsing variables, 1SWASP J221937.28+441703.2 (i.e., V* BS Lac) and 1SWASP J230311.84+423909.2 (i.e., GSC 03224 -02811 ), in phase space. The top panel shows the original SuperWASP light curves, while the bottom panel shows the light curves detrended by the SuperWASP team (Collier Cameron et al. 2006). Other properties of the plots are the same as those of Figure 6, except for the SIMBAD object identifications.
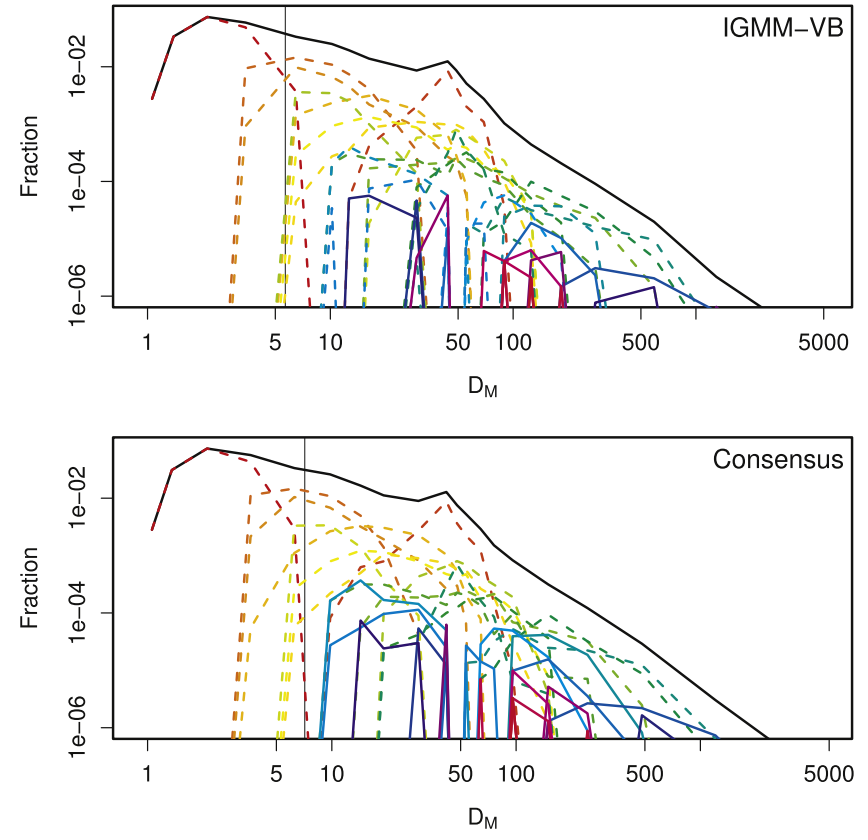

Figure 10. Distributions of $D_{M}$ with respect to the largest cluster in the IGMMVB (top) and consensus (bottom) clustering results. The gross distribution (black) is mainly explained by the light curves included in the large clusters (dashed lines) that comprise more than $10 \%$ of the data. Solid color lines represent the light curves forming small clusters that include less than $10 \%$ of the data. The $99.9 \%$ cut of the $D_{M}$ from the largest cluster is shown as the vertical line.

et al. 2000), VSX catalog (Watson 2006), IRAS point source catalog (Helou \& Walker 1988), IRAS faint-source catalog (Moshir et al. 1990), AKARI/IRC All-sky Point Source Catalog (Version 1.0; Ishihara et al. 2010a, 2010b), AKARI/FIS All-sky Survey Bright Source Catalog (Version 1.0; Yamamura et al. 2010), AllWISE source catalog from the WISE observations (Wright et al. 2010), 2MASS All-sky Catalog of Point Sources (Skrutskie et al. 2006), UKIDSS-DR9 Large Area Survey (Lawrence et al. 2007, 2013), SDSS DR 10 (Ahn et al. 2014), GALEX GR6/7 Data Release, ${ }^{14}$ GALEX BCS (Bianchi, Conti, Shiao) All-sky Imaging Survey (Bianchi et al. 2014), 1SWXRT

\footnotetext{
${ }^{14}$ http://galex.stsci.edu/
}

catalog (D'Elia et al. 2013), and 1SXPS catalog (Evans et al. 2014). The cross-matching is conducted with a $6^{\prime \prime}$ search radius for the given SuperWASP coordinates. We also provide links of queried objects to raw light curves serviced by the NASA Exoplanet Archive ${ }^{15}$ and the CERIT Scientific Cloud. ${ }^{16}$

Cross-matching to other catalogs is mostly useful for identifying interesting variable candidates depending on their spectral types or colors as well as rare spectral energy distributions over other wavelengths. For example, the SDSS colors are widely used for inferring origins of variability by identifying types of variability based on photometric colors (e.g., Süveges et al. 2012; Gezari et al. 2013). In addition to using binary-like light curves, unusual colors can be used to identify candidate binaries (e.g., Hoard et al. 2007).

\section{Conclusions and Discussion}

We expect that using multiple clustering methods and combining together the results obtained using these methods as a way of performing robust clustering will allow us to discover new interesting variable objects by improving the accessibility of big astronomical time-series data to researchers (see Feigelson \& Babu 2012 for a review). Our analysis of the SuperWASP data explores two potentially powerful applications of machine learning algorithms in astronomy. We consider the computationally cheap IGMM-VB clustering method and the consensus clustering method.

The variability of the bright stars investigated in our papers will be continuously highlighted by future public data releases of several projects such as GAIA (Gaia et al. 2016) and TESS (Ricker et al. 2015). Therefore, we anticipate that our analysis of bright sources will be useful in the joint analysis with the new data. Using these future public data together effectively will demand a robust and fast analysis algorithm that accounts for the different properties of data acquired by multiple extra observations.

The extensive usage of our analysis is still hindered by several problems that need to be resolved by future research. The detection of variability using machine learning algorithms needs to improve its usage of features describing information

\footnotetext{
15 http://exoplanetarchive.ipac.caltech.edu/

16 http://wasp.cerit-sc.cz/
} 


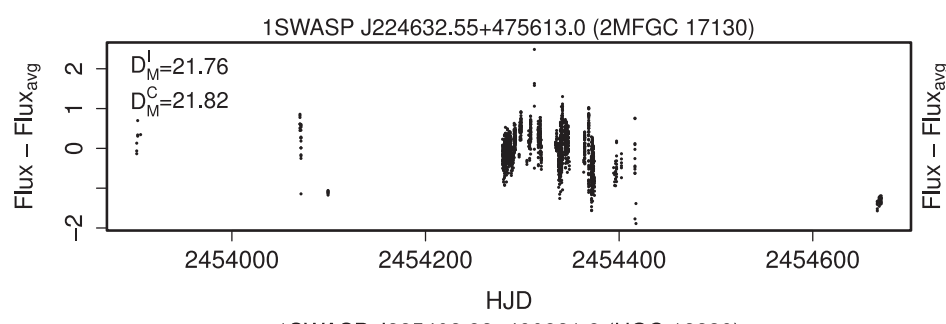

1SWASP J225402.82+490821.8 (UGC 12239)

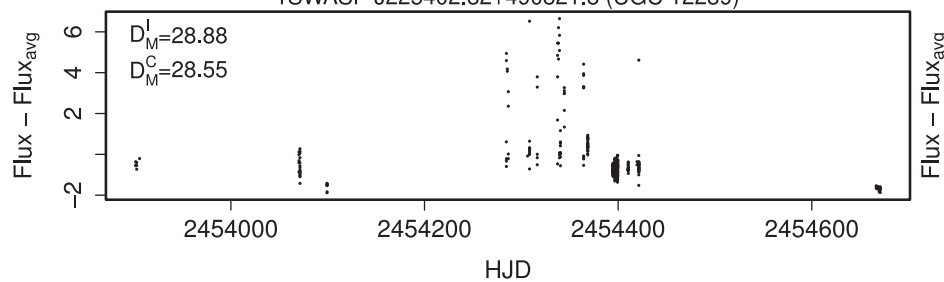

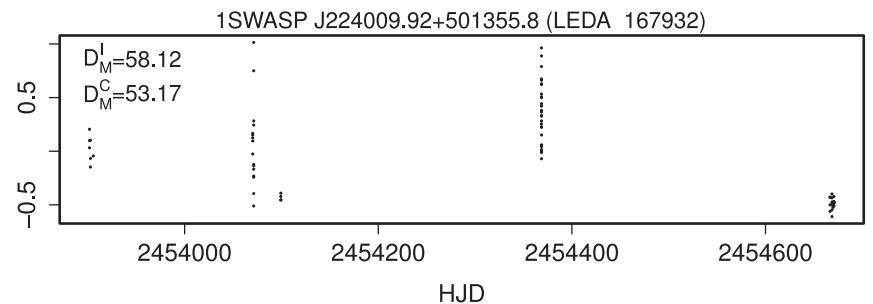

1SWASP J223847.41+474431.3 (2MASX J22384761+4744336)

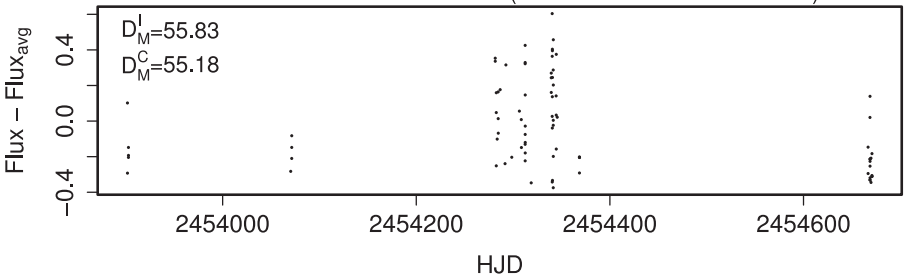

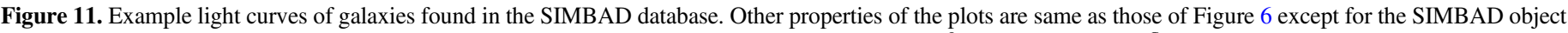

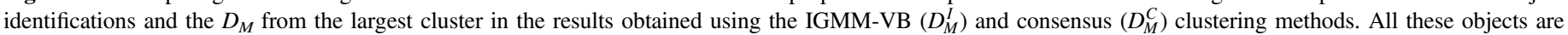
selected as variable candidates when using the $99 \% D_{M}$ cut in both the IGMM-VB and consensus clustering results.

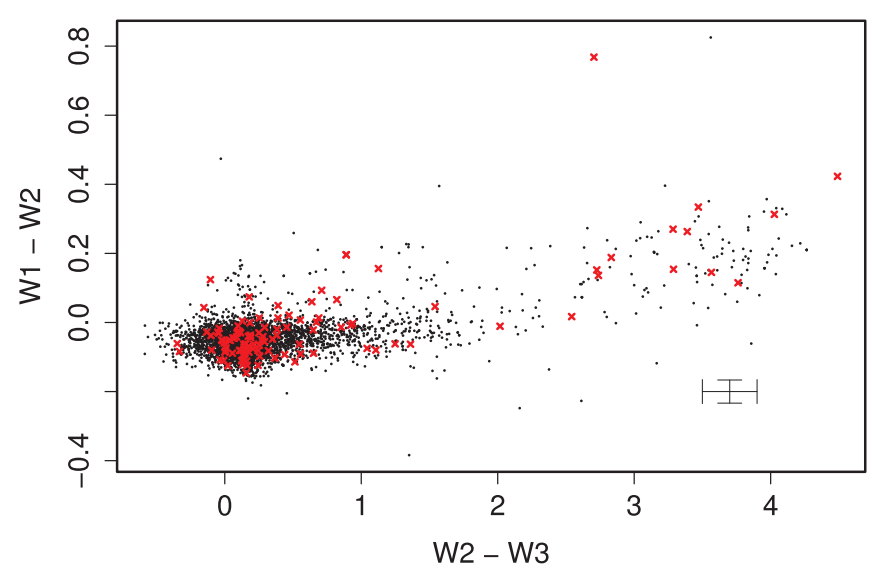

Figure 12. Distribution of WISE colors $W 1-W 2$ and $W 2-W 3$. The diagram shows only the objects with photometric quality flags of A or B (i.e., a flux signal-to-noise ratio $>3$ ) in all three bands (Cutri et al. 2014). The 114 variable candidates found by the IGMM-VB method are shown as crosses. The average of the color errors is represented as a large cross with arrows.

embedded in light curves. It is well known in machine learning research that features such as Table 1 embrace different amounts and types of information and variation in a given data set (Blum \& Langley 1997; Dy \& Brodley 2004). Our research has focused on a quite limited range of features (see Pashchenko et al. 2018 for a larger collection of features). We also do not include machine learning features that can examine the adequacy of specific variability models such as microlensing (e.g., Kim et al. 2018) and transit (e.g., Collier Cameron et al. 2006). Moreover, there is no well-defined, systematic way to represent the complete information stored in light curves from various observations by transformation to the feature space (Nun et al. 2015; Djorgovski et al. 2016; Kim \& Bailer-Jones 2016). In particular, the variation caused by random noise in light curves is considered when estimating the features in our experiments as described in Table 1. However, learning algorithms incorporating the effects of the measurement uncertainty (see Gebru et al. 2016 for an example) might help us exploit a broad range of variability features that do not explicitly take into account random noise in light curves. As a way to improve the reliability of variability detection, future research needs to consider the application of semi-supervised learning methods (Chapelle et al. 2010). The number of known variable sources among bright objects is quite small as found in the GCVS catalog (Samus' et al. 2017), which includes only about 50,000 variable stars. However, the number of known variable stars will increase dramatically as projects such as GAIA (Holl et al. 2018) and TESS discover and classify new bright variable objects. Semi-supervised learning methods will make the variability detection problem similar to a classification problem of variable and non-variable objects by using the small fraction of labeled data (i.e., known variable objects) as a guide for determining boundaries between variable and nonvariable sources (possibly with variability taxonomy classification) in the multidimensional feature space (see Peikari et al. 2018; Sakai et al. 2018, for examples).

The effects of detrending light curves have not been investigated in this paper intentionally because they significantly depend on the detrending implementation. If detrending light curves works perfectly and the corrected light curves do not have any systematic patterns, there must be a single dominant cluster representing non-variable light curves in clustering results. In this case, minor clusters include genuinely variable light curves. Therefore, clustering results can be used to evaluate the performance of different detrending processes, identifying side effects of detrending methods on light curves. We plan to explore this possibility in the future.

The cluster ensemble method used in our paper is not the only available way to combine results acquired by multiple different clustering results. There are numerous ways of ensembling multiple clustering results in machine learning research (see Ghosh \& Acharya 2011 for other possible methods). With increasing computing power and open access to other clustering/classification results, we expect ensemble methods to become increasingly popular. Moreover, ensemble methods can be used to integrate inferences derived for different light curves of the same objects. For example, if SuperWASP objects in our analysis are included in other observations with useful light curves, it will be possible to combine the clustering results acquired by analyzing two different sets of observed light curves, improving efficiency and reliability in detecting variable objects. 

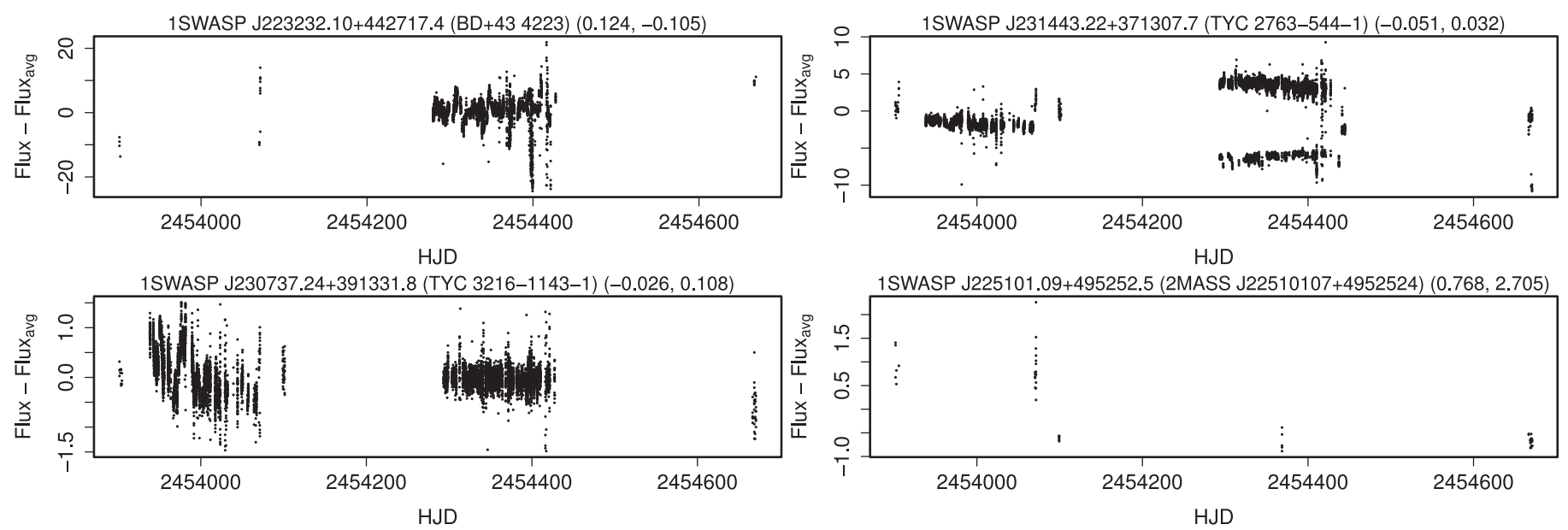

Figure 13. Example light curves of WISE sources as variable candidates. Other properties of the plots are the same as those of Figure 6, except for the SIMBAD object identifications and $(W 1-W 2, W 2-W 3)$ colors. Except for 1SWASP J225101.09+495252.5, the colors of the example objects are close to the typical stellar color around 0 .

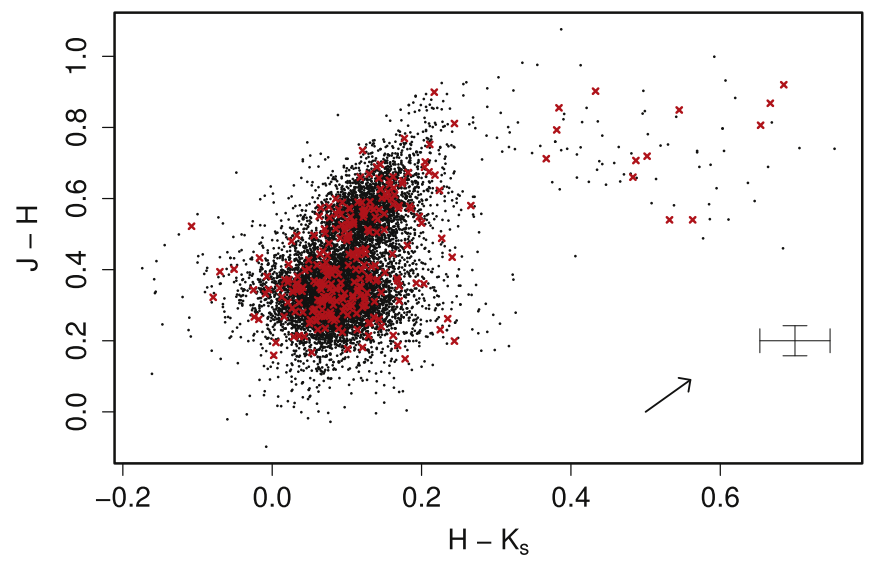

Figure 14. Distribution of 2MASS colors $J-H$ and $H-K_{s}$. The diagram shows only 9329 objects with flags of good photometry (quality flag Qflg $=A$ ) in all three bands (Cutri et al. 2003). The red crosses correspond to 283 variable candidates as members of minor groups found by the IGMM-VB method, as described in Section 4.1. The average of color errors is represented as a large cross with arrows. The one-headed arrow denotes a reddening vector for $A_{r}=1$ in the SDSS $r$-band (Davenport et al. 2014).

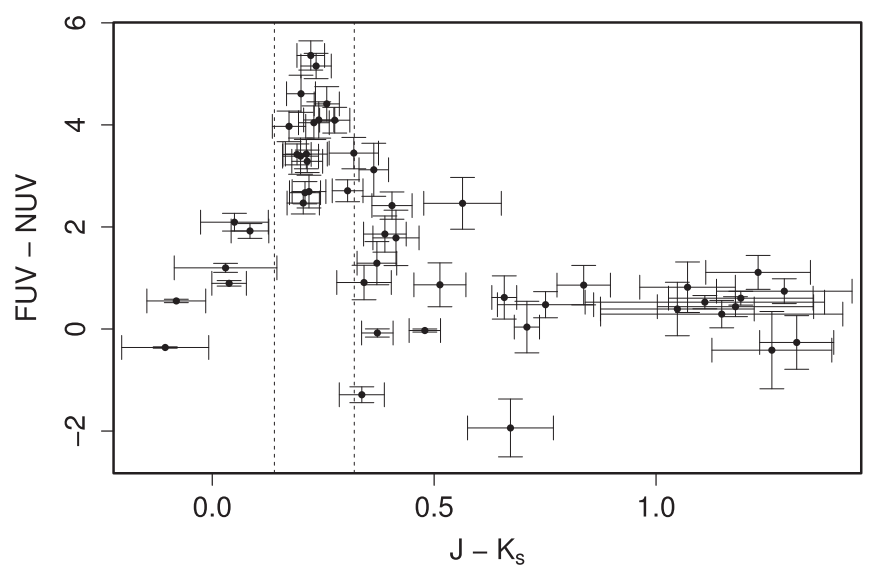

Figure 15. Distribution of GALEX and 2MASS colors $J-K_{s}$ and FUV - NUV. The diagram displays colors of only 48 objects with good GALEX and 2MASS photometric measurements in the relevant bands, among the 10,026 example light curves. The dashed line outlines the instability strip of RR Lyrae stars ranging from 6100 to $7200 \mathrm{~K}$ (Borissova et al. 2009). The FUV - NUV color of most objects is consistent with this temperature range (Smith et al. 2014).
We thank Oliver Butters for helping us access the SuperWASP data. We also thank the referee for careful reading and comments. We would like to acknowledge the support from KISTI (Korea Institute of Science Technology Information) under the contract of the commissioned research project, Massive Astronomical Data Applications of Cloud computation (KISTI-P11020), with Jaegyoon Hahm, Yong-Hwan Jung, Joon-Weon Yoon, Jae-Hyuck Kwak, and Joo Hyun Kim as technical supporters in the KISTI. S.-W.C. acknowledges the support from the KASI (Korea Astronomy and Space Science Institute)-Yonsei research collaboration program for the frontiers of astronomy and space science (2016-1-843-00). Parts of this research were also conducted by the Australian Research Council Centre of Excellence for All-sky Astrophysics (CAASTRO), through project number CE110001020. We acknowledge the WASP consortium which comprises the University of Cambridge, Keele University, University of Leicester, The Open University, The Queens University Belfast, St. Andrews University, and the Isaac Newton Group. Funding for WASP comes from the consortium universities and from the UK's Science and Technology Facilities Council. This research has made use of the SIMBAD database, operated at CDS, Strasbourg, France. This research has also made use of the NASA/ IPAC Infrared Science Archive, which is operated by the Jet Propulsion Laboratory, California Institute of Technology, under contract with the National Aeronautics and Space Administration. This research is based on observations with AKARI, a JAXA project with the participation of ESA. This publication makes use of data products from the Wide-field Infrared Survey Explorer, which is a joint project of the University of California, Los Angeles, and the Jet Propulsion Laboratory/California Institute of Technology, and NEOWISE, which is a project of the Jet Propulsion Laboratory/California Institute of Technology. WISE and NEOWISE are funded by the National Aeronautics and Space Administration. This publication also makes use of data products from the Two Micron All Sky Survey, which is a joint project of the University of Massachusetts and the Infrared Processing and Analysis Center/California Institute of Technology, funded by the National Aeronautics and Space Administration and the National Science Foundation. This work is based in part on data obtained as part of the UKIRT Infrared Deep Sky Survey. Funding for SDSS-III has been 

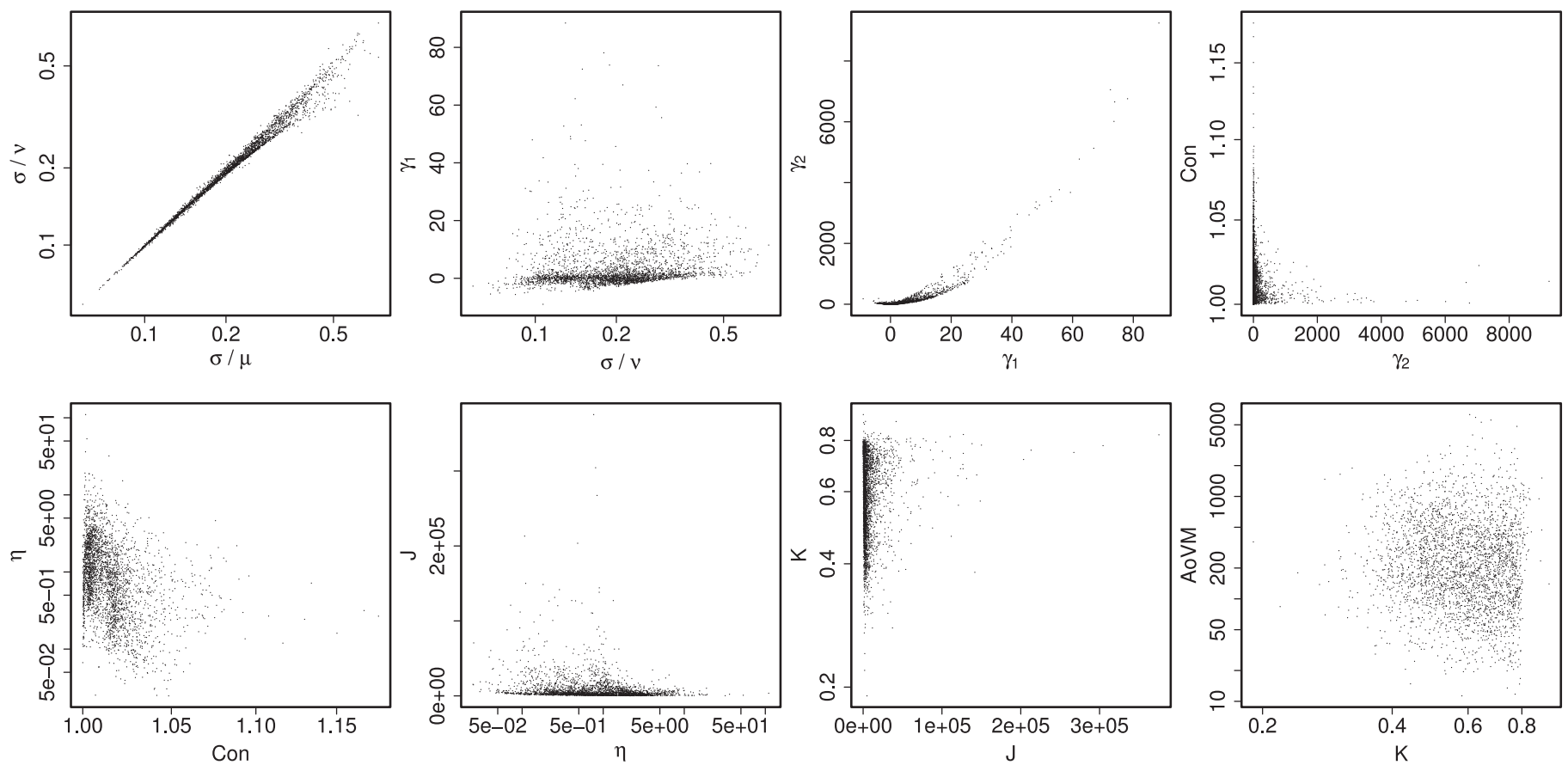

Figure 16. Distributions of variability indices for 3000 light curves in the example analysis presented in Section 3.2 .
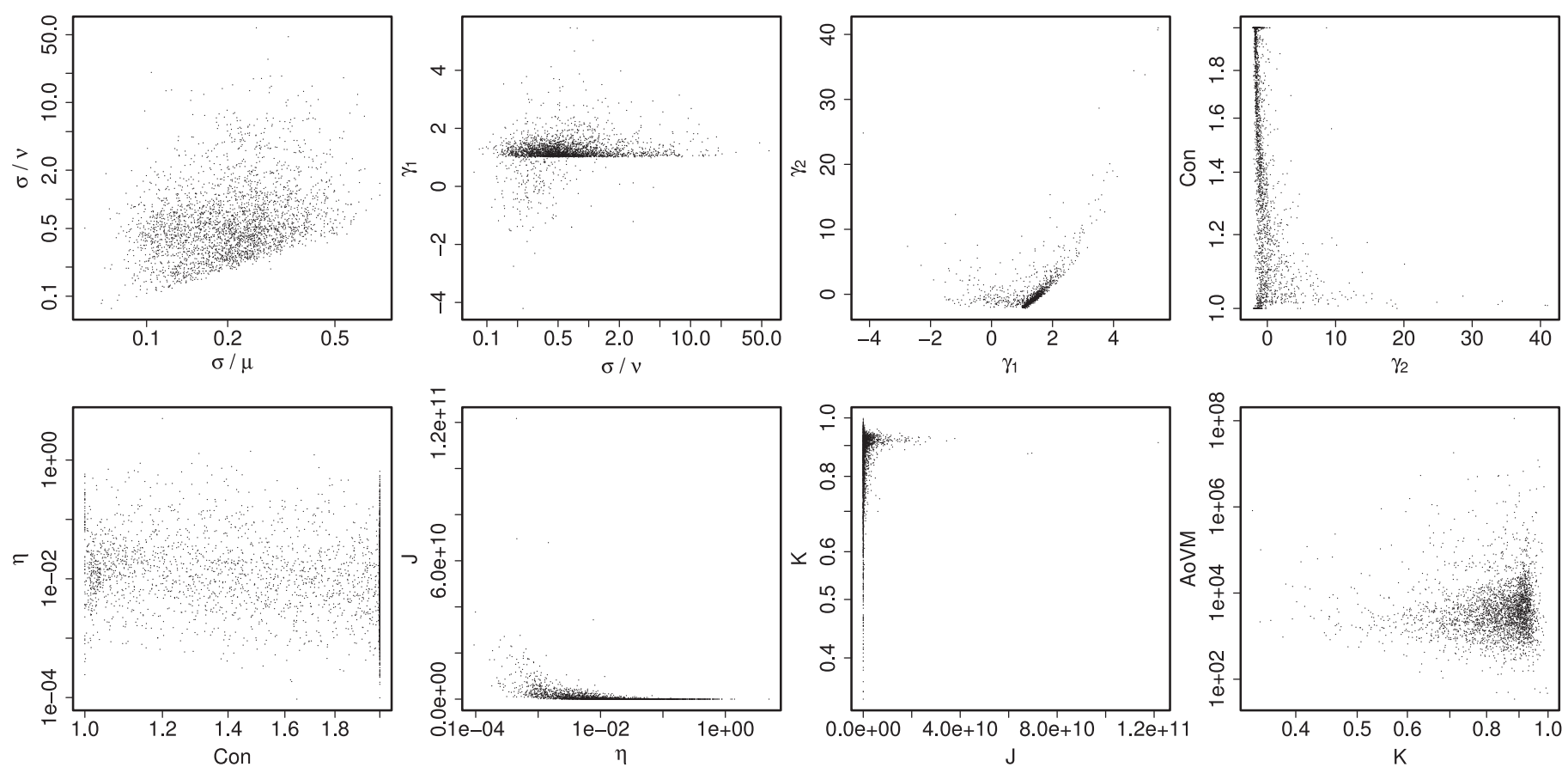

Figure 17. Distributions of variability indices for 3000 simulated red-noise light curves.

provided by the Alfred P. Sloan Foundation, the Participating Institutions, the National Science Foundation, and the U.S. Department of Energy Office of Science. The SDSS-III web site is http://www.sdss3.org/. SDSS-III is managed by the Astrophysical Research Consortium for the Participating Institutions of the SDSS-III Collaboration including the University of Arizona, the Brazilian Participation Group, Brookhaven National Laboratory, Carnegie Mellon University, University of Florida, the French Participation Group, the German Participation Group, Harvard University, the Instituto de Astrofisica de Canarias, the Michigan State/Notre Dame/ JINA Participation Group, Johns Hopkins University, Lawrence Berkeley National Laboratory, Max Planck Institute for Astrophysics, Max Planck Institute for Extraterrestrial Physics, New Mexico State University, New York University, Ohio State University, Pennsylvania State University, University of Portsmouth, Princeton University, the Spanish Participation Group, University of Tokyo, University of Utah, Vanderbilt University, University of Virginia, University of Washington, and Yale University. 

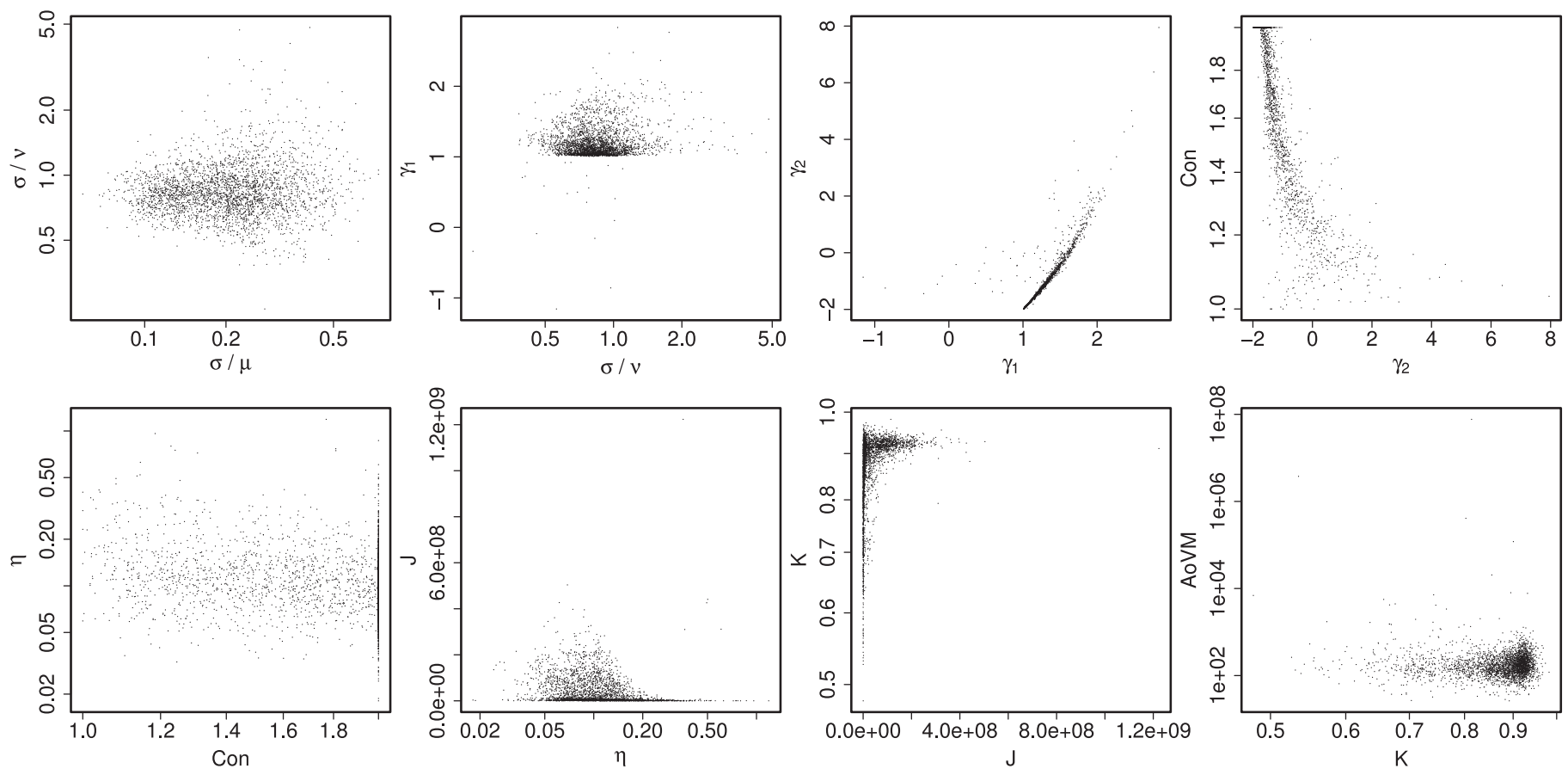

Figure 18. Distributions of variability indices for 3000 simulated pink-noise light curves.
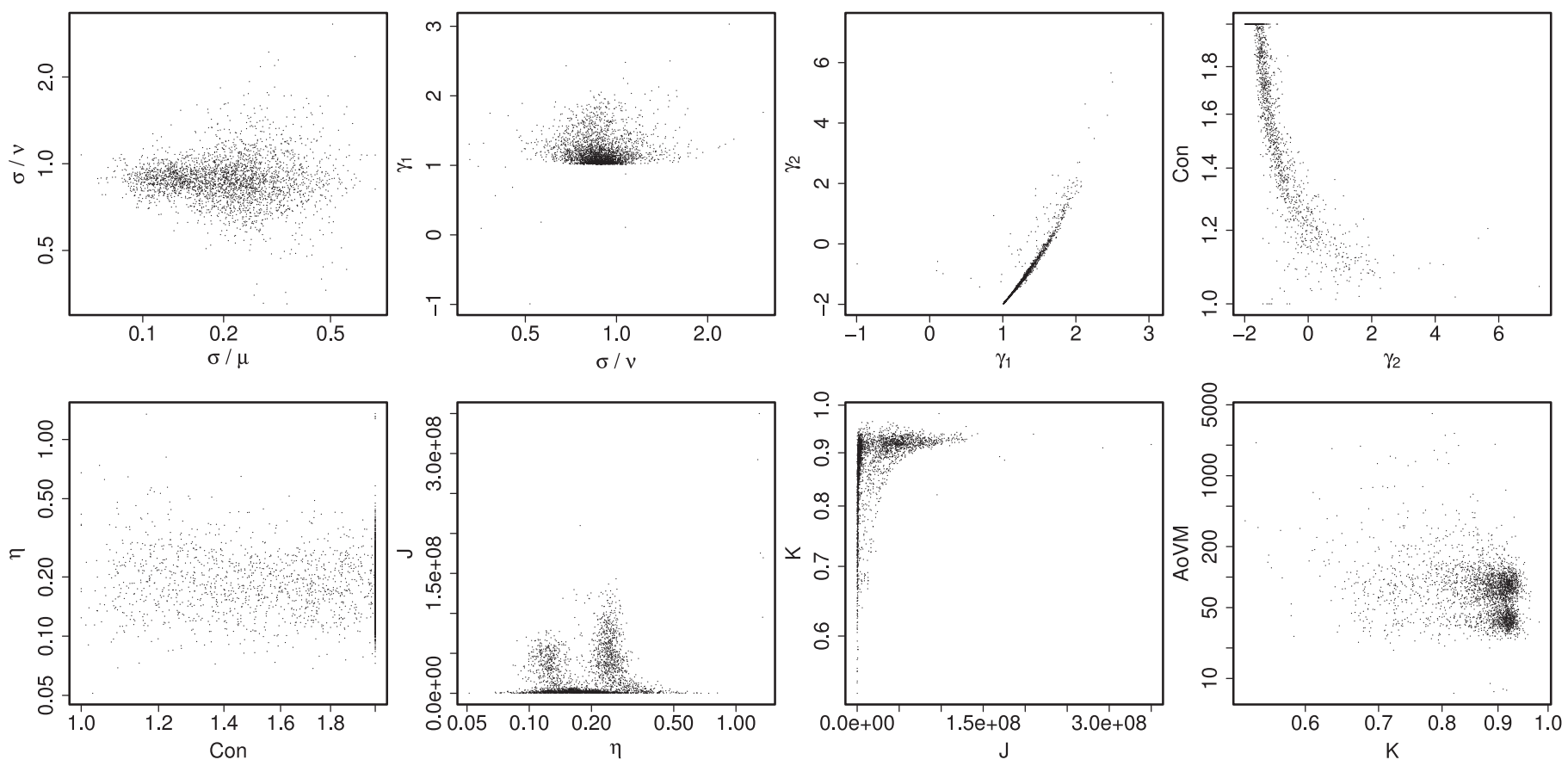

Figure 19. Distributions of variability indices for 3000 simulated white-noise light curves.

\section{Appendix A \\ Interpretation of Variability Indices}

We present distributions of variability indices, which we adopt as machine learning features for clustering analysis and variability detection, in three cases of red, pink, and white noises, which have power-law power spectra (Timmer \& Koenig 1995). The distributions help readers to understand how the variability indices appear for some well-known systematic patterns that can be found in light curves, although there are other factors such as temporal sampling patterns and non-random systematic signal.
Mock light curves describing red, pink, and white noises are generated and have the same sampling epochs and $\sigma / \mu$ ratio, which is matched by scaling $\mu$ in terms of either adding or subtracting fluxes for simulated light curves, with the original SuperWASP light curves. Figures 16-19 show the distribution of the variability indices for 3000 light curves including the original SuperWASP light curves, which are included in the example analysis of Section 3.2, and the simulated light curves.

Some variability indices show substantially different distributions between the raw SuperWASP light curves and the simulated noise light curves. For example, the joint distribution 

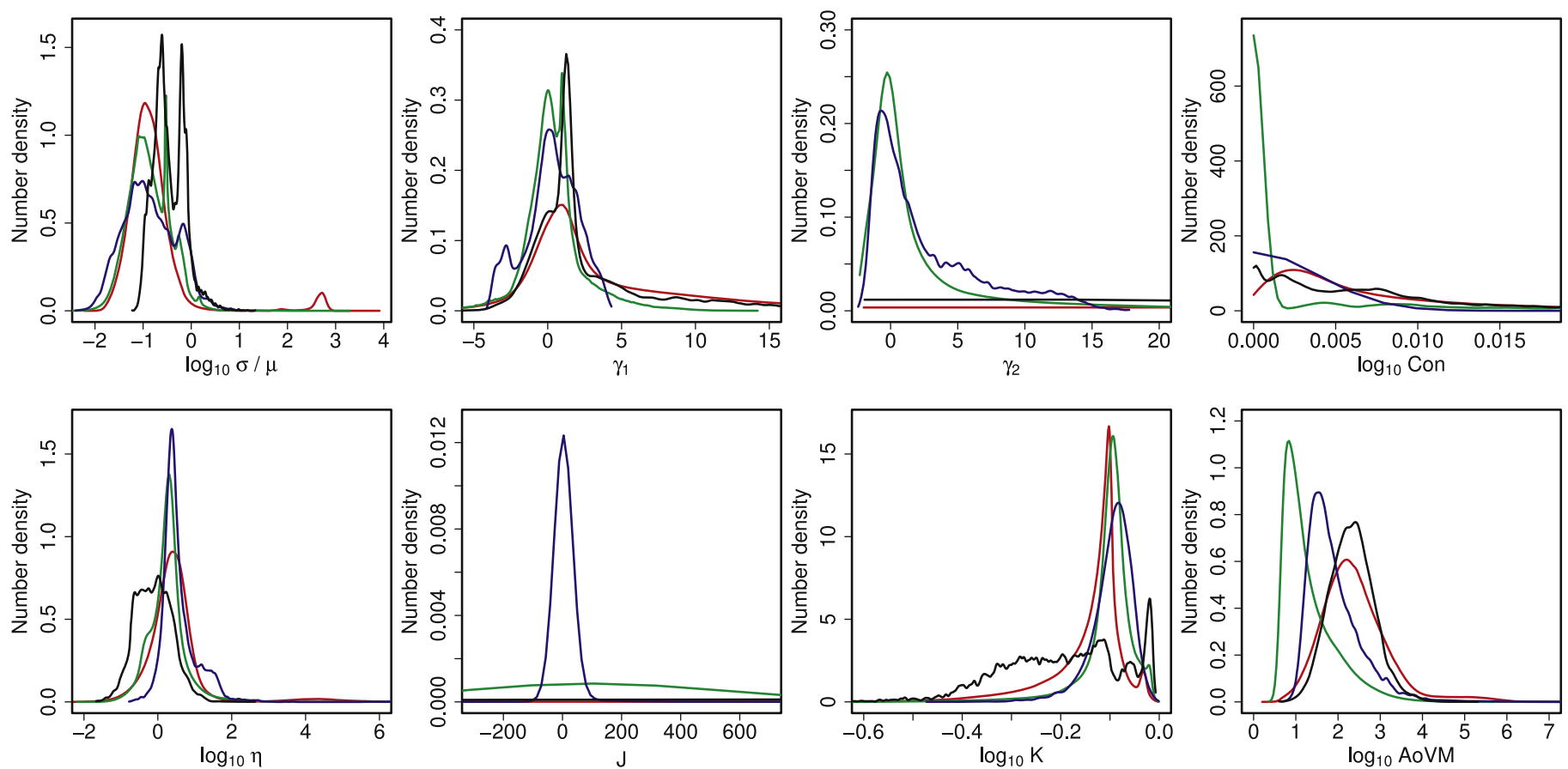

Figure 20. Distribution of variability indices with respect to the number of observations in the SuperWASP light curves. The number density distributions derived using a kernel density estimation are presented for the three subsets of different numbers of observations: 11-15 (blue), 16-200 (green), and >200 (red). The distribution of the data presented in Section 3.2 is also presented in black. $\gamma_{2}$ and $J$ have a wide range for light curves with more than 200 observation epochs per light curve and the example data set (black), looking similar to a uniform distribution.
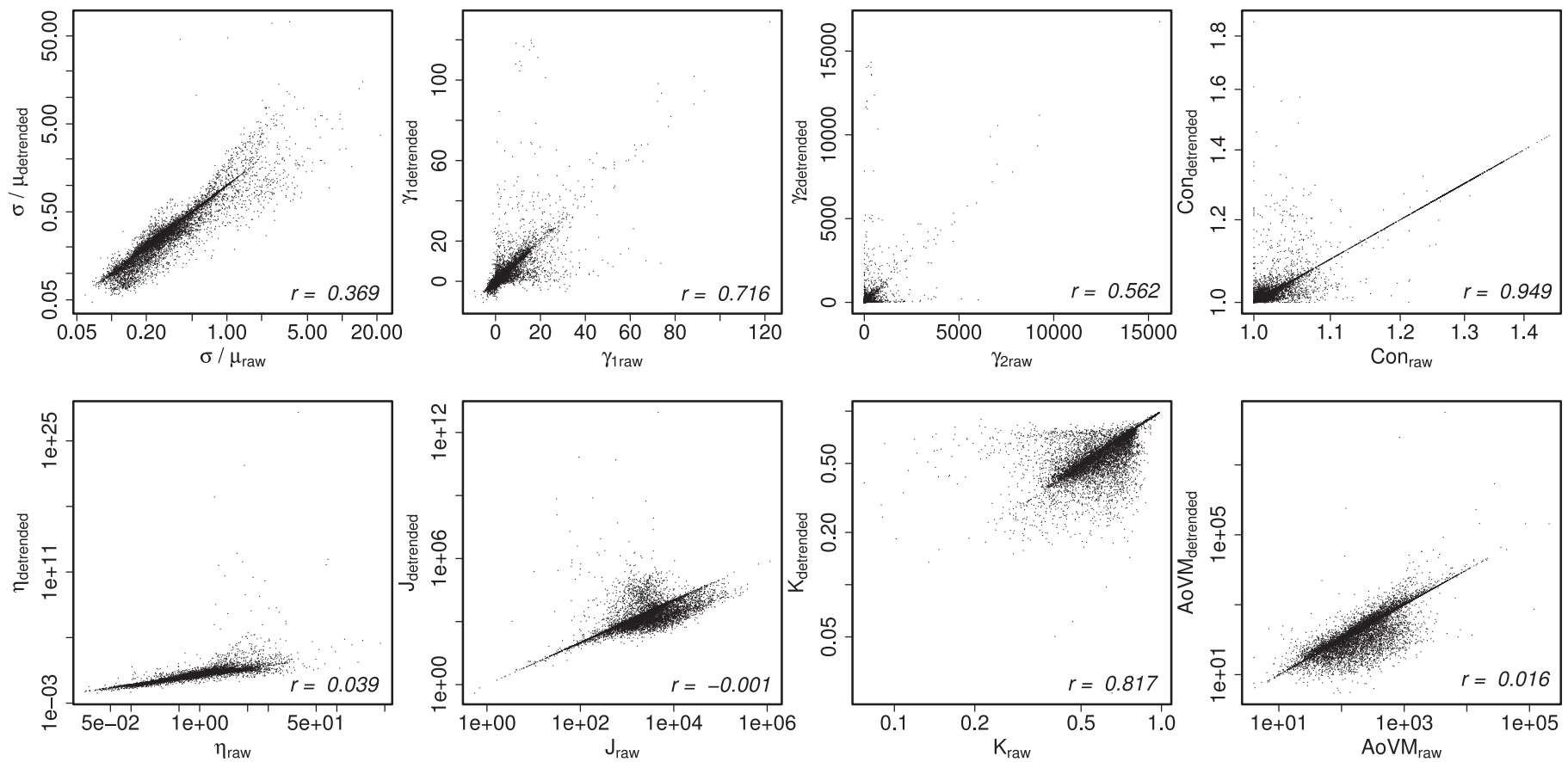

Figure 21. Distribution of variability indices from the raw calibrated light curves ( $x$-axis) and the detrended light curves ( $y$-axis) of the example analysis data presented in Section 3.2. Each plot presents a Pearson correlation coefficient of the variability indices between the two different cases.

of $\sigma / \mu$ and $\sigma / \nu$ in the simulated light curves does not follow the same trend as that in the SuperWASP light curves. The value ranges of $\gamma_{1}$ and $\gamma_{2}$ in the simulated light curves are also quite different from those in the original SuperWASP light curves. These examples show that the SuperWASP light curves are not completely dominated by a single simple pattern such as red, pink, and white noises.
We also show the distribution of variability indices with respect to the number of data points in the light curves. If light curves are made of fluxes sampled from stationary distributions, a large number of observations let variability indices have a well-estimated representative value (i.e., non-biased estimation) for the stationary distributions. We note that interpreting these distributions of variability indices is not 
straightforward in cases where several non-stationary effects are entangled together as expected in most ground-based observations like the SuperWASP. Therefore, it is necessary to use light curves of similar sizes as input data for clustering as we extract sets of light curves over a similar temporal coverage in Section 2.

Figure 20 presents the distribution of variability indices except for $\sigma / \nu$, which is quite similar to $\sigma / \mu$, with respect to the number of observation epochs in the SuperWASP light curves. Several indices such as AoVM show obvious variations as a function of observation epochs.

As we discuss in Section 7, we expect that the quality of detrended light curves might be evaluated by inspecting clustering results of detrended light curves and comparing their distributions with those of clustering raw light curves. This possibility can be checked in the comparison of the variability indices between the raw light curves and the detrended light curves (see Figure 21). The difference in $J$ seems significant in this check as measured by the negative Pearson correlation coefficient. The distributions of $\eta$ and AoVM also imply that the results of clustering the detrended light curves can be very different from what we find with the raw light curves due to the large changes in their detrended light curves.

\section{Appendix B \\ Mixture Distribution Models and Implementations of Statistical Inference}

In this appendix, we summarize the key components of mixture distribution models and clustering. The overall introduction to all of these models can be found in well-known machine learning literature such as Murphy (2012).

The IGMM-MCMC method, which was used in Papers I and II, uses the Dirichlet Process (DP) infinite Gaussian mixture model (see Magorrian 2014 for an example in astrophysics). See Papers I and II for the basic components of the model. The models are described conceptually by the following as shown in Blei \& Jordan (2006):

$$
\begin{gathered}
\alpha \sim \operatorname{Gamma}\left(s_{1}, s_{2}\right), \\
G \mid \alpha, G_{0} \sim \operatorname{DP}\left(\alpha, G_{0}\right), \\
\eta_{i} \mid G \sim G, \\
X_{i} \mid \eta_{i} \sim \operatorname{Normal}\left(x_{i} \mid \eta_{i}\right),
\end{gathered}
$$

where $s_{1}$ and $s_{2}$ are the shape and rate parameters of the Gamma distribution as constants, respectively, DP is the Dirichlet process with the base distribution $G_{0}$ (i.e., normal inverse Wishart distribution in this paper), and $\eta$ is the parameter of the multivariate normal distribution (i.e., mean and covariance). When we use the allocation variable $z$ as a latent variable and a stick-breaking process for the DP prior (Hastie et al. 2014), our model can be expressed as follows:

$$
\begin{gathered}
\alpha \sim \operatorname{Gamma}\left(s_{1}, s_{2}\right), \\
V_{l} \sim \operatorname{Beta}(1, \alpha) \text { and } V_{1}=w_{1}, \\
w_{k}=V_{k} \prod_{l<k}\left(1-V_{l}\right), \\
\sigma_{k} \sim \operatorname{Inverse}-\operatorname{Wishart}\left(\lambda_{0}, v_{0}\right), \\
\mu_{k} \sim \operatorname{Normal}\left(\mu_{0}, \sigma_{k} / \kappa_{0}\right),
\end{gathered}
$$

where $P\left(z_{i}=c \mid w\right)=w_{c}$ and other symbols of the probability distribution parameters correspond to hyperparameters.

There are two important differences between the implementation of the IGMM-MCMC in the current paper and that used in Papers I and II, in addition to using a different MCMC sampler and exploiting the better implementation of the models by Liverani et al. (2015) and Hastie et al. (2014). First, in this paper, the concentration parameter (or the so-called scaling parameter) $\alpha$ of the DP (Escobar \& West 1995) is modeled using the Gamma distribution, which is characterized by shape and rate parameters, as a prior on it in this paper, while it was a constant in our previous papers. This change makes the current model more flexible and adequate for treating complex data. We use 2 and 1 as values of the shape and rate parameters in the Gamma distribution prior. Second, while the assignment of cluster membership is determined by the point estimation of a mode in the posterior distribution in the previous papers, here we use a posterior similarity matrix $S$ that is an averaged similarity matrix of cluster membership for a pair of data in the MCMC samples (see Lau \& Green 2007 for a discussion), producing a dissimilarity matrix that is equal to $1-S$. In short, the element $S_{i j}$ represents the posterior probability that two light curves $i$ and $j$ are found in the same cluster in the MCMC samples. From this dissimilarity matrix, we find the best partitioning around medoid, which has the largest average silhouette width (see Reynolds et al. 2006 for a full explanation).

The IGMM-VB method uses a variational approximation, which is much faster than inference based on MCMC sampling in the IGMM-MCMC model. Our model using the variational approximation uses the implementation presented by Blei \& Jordan (2006). The posterior distribution is found in an iterative process of finding the approximate distribution.

The model corresponding to the GMM-EM method consists of maximum-likelihood inference with finite Gaussian mixture components that are described by Equation (1) for a fixed number of components $K$. In order to estimate the model parameters by maximizing the likelihood, we adopt a typical approach using a hidden variable, which corresponds to $z$ in the model of IGMM, and the EM algorithm because of the fact that there is no closed form solution for maximum likelihood. When $\theta$ represents the parameters $\mu_{k}$ and $\Sigma_{k}$ that should be determined in Equation (1), the M-step in the $t$ th EM iteration is

$$
\theta^{t+1}=\underset{\theta}{\operatorname{argmax}} E_{z}\left[\log \left(p\left(\boldsymbol{x}, z \mid \theta^{t}\right)\right)\right],
$$

and the E-step is

$$
E_{z}\left(z_{i k} \mid \theta^{t}\right)=p\left(k \mid x_{i}, \theta^{t}\right),
$$

where $i$ is the index of the data point. The number of mixture components in the best model is chosen by using the Bayesian information criterion that is $-2 \times \log ($ Likelihood $)+($ the number of parameters $) \times \log ($ the size of data).

\section{References}

Ahn, C. P., Alexandroff, R., Allende Prieto, C., et al. 2014, ApJS, 211, 17 Alcock, C., Allsman, R. A., Axelrod, T. S., et al. 1993, in ASP Conf. Ser. 43, Sky Surveys. Protostars to Protogalaxies, ed. B. T. Soifer (San Francisco, CA: ASP), 291

Antoniak, C. E. 1974, AnSta, 2, 1152

Bhat, P. C. 2011, ARNPS, 61, 281

Bianchi, L. \& GALEX Team 1999, MmSAI, 70, 365 
Bianchi, L., Conti, A., \& Shiao, B. 2014, AdSpR, 53, 900

Bianchi, L., Rodriguez-Merino, L., Viton, M., et al. 2007, ApJS, 173, 659

Bishop, C. M. 2007, Pattern Recognition and Machine Learning (1st ed; Berlin: Springer)

Blei, D. M., \& Jordan, M. I. 2006, BayAn, 1, 121

Blum, A. L., \& Langley, P. 1997, Artificial Intelligence, 97, 245

Borissova, J., Rejkuba, M., Minniti, D., Catelan, M., \& Ivanov, V. D. 2009, A\&A, 502, 505

Brink, H., Richards, J. W., Poznanski, D., et al. 2013, MNRAS, 435, 1047

Butters, O. W., West, R. G., Anderson, D. R., et al. 2010, A\&A, 520, L10

Cao, G., \& West, M. 1996, Biometrics, 52, 1334

Chapelle, O., Schlkopf, B., \& Zien, A. 2010, Semi-Supervised Learning (1st ed.; Cambridge, MA: MIT Press)

Chaurasia, A., \& Harel, O. 2013, EJSta, 7, 2762

Cioni, M.-R. L., Girardi, L., Moretti, M. I., et al. 2014, A\&A, 562, A32

Clegg, P. E. 1980, PhyS, 21, 678

Collier Cameron, A., Pollacco, D., Street, R. A., et al. 2006, MNRAS, 373, 799

Cutri, R. M., Skrutskie, M. F., van Dyk, S., et al. 2003, yCat, 2246, 0

Cutri, R. M., et al. 2014, yCat, 2328, 0

Davenport, J. R. A., Ivezić, Ž., Becker, A. C., et al. 2014, MNRAS, 440, 3430

Davies, D. L., \& Bouldin, D. W. 1979, ITPAM, PAMI-1, 224

D’Elia, V., Perri, M., Puccetti, S., et al. 2013, A\&A, 551, A142

Djorgovski, S., Graham, M., Donalek, C., et al. 2016, Future Generation Computer Systems, 59, 95

Drake, A. J., Djorgovski, S. G., Mahabal, A., et al. 2009, ApJ, 696, 870

Dunn, J. C. 1973, Journal of Cybernetics, 3, 32

Dy, J. G., \& Brodley, C. E. 2004, J. Mach. Learn. Res., 5, 845

Edelson, R., \& Malkan, M. 2012, ApJ, 751, 52

Escobar, M. D., \& West, M. 1995, J. Am. Stat. Assoc., 90, 577

Evans, P. A., Osborne, J. P., Beardmore, A. P., et al. 2014, ApJS, 210, 8

Feigelson, E. D., \& Babu, G. J. 2012, Significance, 9, 22

Ferguson, T. S. 1973, AnSta, 1, 209

Filkov, V., \& Skiena, S. 2003, in Proc. 15th IEEE Int. Conf. Tools with Artificial Intelligence (New York: IEEE), 418

Fowlkes, E. B., \& Mallows, C. L. 1983, J. Am. Stat. Assoc., 78, 553

Gaia, C., Prusti, T., de Bruijne, J. H. J., et al. 2016, A\&A, 595, A1

Gebru, I. D., Alameda-Pineda, X., Forbes, F., \& Horaud, R. 2016, ITPAM, 38, 2402

Gezari, S., Martin, D. C., Forster, K., et al. 2013, ApJ, 766, 60

Ghosh, J., \& Acharya, A. 2011, Wiley Interdisciplinary Reviews: Data Mining and Knowledge Discovery, 1, 305

Goder, A., \& Filkov, V. 2008, in 2008 Proc. of the Tenth Workshop on Algorithm Engineering and Experiments (ALENEX) (Philadelphia, PA: SIAM), 109

Grubbs, F. E. 1969, Technometrics, 11, 1

Habibagahi, H., \& Pratschke, J. L. 1972, The Review of Economics and Statistics, 54, 179

Hahm, J., Kwon, O.-K., Kim, S., et al. 2012, in Embedded and Multimedia Computing Technology and Service, ed. J. J. J. H. Park et al. (The Netherlands: Springer), 493

Hainline, K. N., Hickox, R. C., Carroll, C. M., et al. 2014, ApJ, 795, 124

Hastie, D., Liverani, S., \& Richardson, S. 2014, Statistics and Computing, 25,1023

Hebb, L., Stempels, H. C., Aigrain, S., et al. 2010, A\&A, 522, A37

Helou, G., \& Walker, D. W. 1988, iras, 7, 1

Hoard, D. W., Wachter, S., Sturch, L. K., et al. 2007, AJ, 134, 26

Holl, B., Audard, M., Nienartowicz, K., et al. 2018, arXiv:1804.09373

Hubert, L., \& Arabie, P. 1985, Journal of Classification, 2, 193

Ishihara, D., Onaka, T., Kataza, H., et al. 2010a, yCat, 2297, 0

Ishihara, D., Onaka, T., Kataza, H., et al. 2010b, A\&A, 514, A1

Ivezic, Z., Tyson, J. A., Acosta, E., et al. 2008, arXiv:0805.2366

Jain, A. K. 2010, PaReL, 31, 651

Jain, A. K., Topchy, A., Law, M. H. C., \& Buhmann, J. M. 2004, in 17th Int. Conf. (ICPR'04) 1, Proc. of the Pattern Recognition (Washington, DC: IEEE Computer Society), 260

Jarrett, T. H., Cohen, M., Masci, F., et al. 2011, ApJ, 735, 112

Joanes, D. N., \& Gill, C. A. 1998, Journal of the Royal Statistical Society: Series D (The Statistician), 47, 183

Jogesh Babu, G., Mahabal, A., Djorgovski, S. G., \& Williams, R. 2008, StMet, 5, 299

Kane, S. R., Collier Cameron, A., Horne, K., et al. 2004, MNRAS, 353, 689

Kim, D.-J., Kim, H.-W., Hwang, K.-H., et al. 2018, AJ, 155, 76

Kim, D.-W., \& Bailer-Jones, C. A. L. 2016, A\&A, 587, A18

Kleinmann, S. G. 1992, in ASP Conf. Ser. 34, Robotic Telescopes in the 1990s, ed. A. V. Filippenko (San Francisco, CA: ASP), 203

Krisciunas, K., Margon, B., \& Szkody, P. 1998, PASP, 110, 1342
Kwak, J.-H., Yoon, J., Jung, Y., Hahm, J., \& Park, D. 2011, Journal of KISS: Computing Practices, 17, 587

Lau, J. W., \& Green, P. J. 2007, Journal of Computational and Graphical Statistics, 16, 526

Lawrence, A., Warren, S. J., Almaini, O., et al. 2007, MNRAS, 379, 1599

Lawrence, A., Warren, S. J., Almaini, O., et al. 2013, yCat, 2319, 0

Liddle, A. R. 2007, MNRAS, 377, L74

Liverani, S., Hastie, D. I., Azizi, L., Papathomas, M., \& Richardson, S. 2015, Journal of Statistical Software, 64, 1

Magorrian, J. 2014, MNRAS, 437, 2230

Marin, J.-M. 2007, Bayesian Core: A Practical Approach to Computational Bayesian Statistics (Springer Publishing Company, Incorporated)

Marin, J.-M., \& Robert, C. P. 2007, Bayesian Core: a Practical Approach to Computational Bayesian statistics (New York: Springer)

Martin, D. C., Fanson, J., Schiminovich, D., et al. 2005, ApJ, 619, L1

Maulik, U., \& Bandyopadhyay, S. 2002, ITPAM, 24, 1650

McQuillin, R., Evans, A., Wilson, D., et al. 2012, MNRAS, 419, 330

Melnykov, V., Chen, W.-C., \& Maitra, R. 2012, Journal of Statistical Software, 51,1

Moon, T. 1996, ISPM, 13, 47

Moshir, M., et al. 1990, IRAS Faint Source Catalogue, version 2.0, 0

Murakami, H., Baba, H., Barthel, P., et al. 2007, PASJ, 59, 369

Murphy, K. P. 2012, Machine Learning: A Probabilistic Perspective (Cambridge, MA: MIT Press)

Neal, R. M. 2000, Journal of Computational and Graphical Statistics, 9, 249

Nieto-Barajas, L. E., \& Contreras-Cristán, A. 2014, BayAn, 9, 147

Nikutta, R., Hunt-Walker, N., Nenkova, M., Ivezić, Ž., \& Elitzur, M. 2014, MNRAS, 442, 3361

Norton, A. J. 2012, in IAU Symp. 285, New Horizons in Time-Domain Astronomy, ed. E. Griffin, R. Hanisch, \& R. Seaman (Cambridge: Cambridge Univ. Press), 382

Norton, A. J., Payne, S. G., Evans, T., et al. 2011, A\&A, 528, A90

Norton, A. J., Wheatley, P. J., West, R. G., et al. 2007, A\&A, 467, 785

Nun, I., Protopapas, P., Sim, B., et al. 2015, arXiv:1506.00010

Pashchenko, I. N., Sokolovsky, K. V., \& Gavras, P. 2018, MNRAS, 475, 2326

Paunzen, E., Kuba, M., West, R. G., \& Zejda, M. 2014, IBVS, 6090, 1

Peikari, M., Salama, S., Nofech-Mozes, S., \& Martel, A. L. 2018, NatSR, 8, 7193

Pojmanski, G. 1997, AcAau, 47, 467

Pollacco, D. L., Skillen, I., Collier Cameron, A., et al. 2006, PASP, 118, 1407

Pradhan, A. C., Ojha, D. K., Robin, A. C., Ghosh, S. K., \& Vickers, J. J. 2014 A\&A, 565, A33

Price-Whelan, A. M., Agüeros, M. A., Fournier, A. P., et al. 2014, ApJ, 781, 35

Rand, W. M. 1971, J. Am. Stat. Assoc., 66, 846

Rasmussen, C. E. 2000, In Advances in Neural Information Processing Systems, Vol. 12 (Cambridge, MA: MIT Press), 554

Reynolds, A., Richards, G., de la Iglesia, B., \& Rayward-Smith, V. 2006, Journal of Mathematical Modelling and Algorithms, 5, 475

Reynolds, D. 2015, in Gaussian Mixture Models, ed. S. Z. Li \& A. K. Jain (Boston, MA: Springer), 827

Ricker, G. R., Winn, J. N., Vanderspek, R., et al. 2015, JATIS, 1, 014003

Ridgway, S. T., Matheson, T., Mighell, K. J., Olsen, K. A., \& Howell, S. B. 2014, ApJ, 796, 53

Rimoldini, L. 2014, MNRAS, 437, 147

Robert, C. 1996, in Markov Chain Monte Carlo in Practice, ed. W. Gilks, S. Richardson, \& D. Spiegelhalter (US: Springer), 441

Sakai, T., Niu, G., \& Sugiyama, M. 2018, Mach. Learn., 107, 767

Samus', N. N., Kazarovets, E. V., Durlevich, O. V., Kireeva, N. N., \& Pastukhova, E. N. 2017, ARep, 61, 80

Schaffenroth, V., Geier, S., Drechsel, H., et al. 2013, A\&A, 553, A18

Schwarzenberg-Czerny, A. 1989, MNRAS, 241, 153

Schwarzenberg-Czerny, A. 1996, ApJ, 460, L107

Sesar, B., Stuart, J. S., Ivezić, Ž., et al. 2011, AJ, 142, 190

Shin, M.-S., \& Byun, Y.-I. 2004, JKAS, 37, 79

Shin, M.-S., Sekora, M., \& Byun, Y.-I. 2009, MNRAS, 400, 1897

Shin, M.-S., Yi, H., Kim, D.-W., Chang, S.-W., \& Byun, Y.-I. 2012, AJ, 143,65

Skrutskie, M. F., Cutri, R. M., Stiening, R., et al. 2006, AJ, 131, 1163

Smalley, B., Kurtz, D. W., Smith, A. M. S., et al. 2011, A\&A, 535, A3

Smith, M. A., Bianchi, L., \& Shiao, B. 2014, AJ, 147, 159

Stetson, P. B. 1996, PASP, 108, 851

Süveges, M., Sesar, B., Váradi, M., et al. 2012, MNRAS, 424, 2528

Thain, D., Tannenbaum, T., \& Livny, M. 2005, Concurrency and Computation Practice and Experience, 17, 323

Theissen, C. A., \& West, A. A. 2014, ApJ, 794, 146

Thieler, A. M., Backes, M., Fried, R., \& Rhode, W. 2013, Statistical Analysis and Data Mining, 6, 73 
Thomas, N. L., Norton, A. J., Pollacco, D., et al. 2010, A\&A, 514, A30

Timmer, J., \& Koenig, M. 1995, A\&A, 300, 707

Trotta, R. 2008, ConPh, 49, 71

Tzikas, D., Likas, C., \& Galatsanos, N. 2008, ISPM, 25, 131

Udalski, A., Szymanski, M., Kaluzny, J., Kubiak, M., \& Mateo, M. 1992, AcAau, 42, 253

Vendramin, L., Campello, R. J. G. B., \& Hruschka, E. R. 2010, Statistical Analysis and Data Mining, 3, 209

Verbeek, K., Groot, P. J., Scaringi, S., et al. 2014, MNRAS, 438, 2

Wald, A., \& Wolfowitz, J. 1940, Ann. Math. Statist., 11, 147

Watson, C. L. 2006, SASS, 25, 47

Weakliem, D. L. 1999, Sociological Methods and Research, 27, 359

Wenger, M., Ochsenbein, F., Egret, D., et al. 2000, A\&AS, 143, 9
Wiley, K., Connolly, A., Gardner, J., et al. 2011, PASP, 123, 366

Williams, R., Hanisch, R., Szalay, A., \& Plante, R. 2008, IVOA Recommendation: Simple Cone Search Version 1.03, http://www.ivoa.net/documents/REC/ DAL/ConeSearch-20080222.html

Woźniak, P. R., Vestrand, W. T., Akerlof, C. W., et al. 2004, AJ, 127, 2436

Wright, E. L., Eisenhardt, P. R. M., Mainzer, A. K., et al. 2010, AJ, 140,1868

Yamamura, I., Makiuti, S., Ikeda, N., et al. 2010, yCat, 2298, 0

Zheng, H., Kulkarni, S., \& Poor, H. 2011, in 45th Annual Conf. Information Sciences and Systems (CISS) (New York: IEEE), 1

Zhou, Z.-H. 2012, Ensemble Methods: Foundations and Algorithms (1st ed.; London, Boca Raton, FL: Chapman and Hall, CRC Press)

Zucchini, W. 2000, Journal of Mathematical Psychology, 44, 41 